Discussion Papers

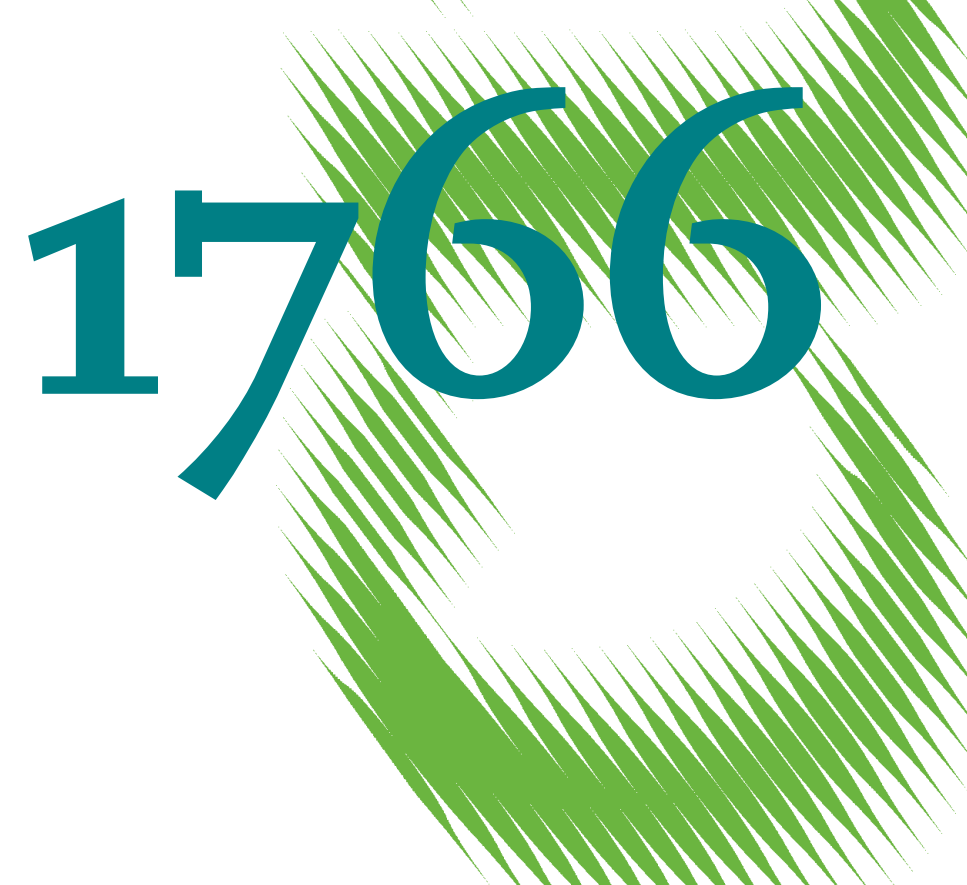

Modeling Coordination between Renewables and Grid

Policies to Mitigate Distribution Grid Constraints Using Residential PV-Battery Systems 
Opinions expressed in this paper are those of the author(s) and do not necessarily reflect views of the institute.

IMPRESSUM

(C) DIW Berlin, 2018

DIW Berlin

German Institute for Economic Research

Mohrenstr. 58

10117 Berlin

Tel. +49 (30) $89789-0$

Fax +49 (30) $89789-200$

http://www.diw.de

ISSN electronic edition 1619-4535

Papers can be downloaded free of charge from the DIW Berlin website:

http://www.diw.de/discussionpapers

Discussion Papers of DIW Berlin are indexed in RePEc and SSRN:

http://ideas.repec.org/s/diw/diwwpp.html

http://www.ssrn.com/link/DIW-Berlin-German-Inst-Econ-Res.html 


\title{
Modeling coordination between renewables and grid: Policies to mitigate distribution grid constraints using residential PV-battery systems
}

\author{
Paul Neetzow ${ }^{\mathrm{a}}$, Roman Mendelevitch ${ }^{\mathrm{a}, *}$, Sauleh Siddiqui ${ }^{\mathrm{b}, \mathrm{c}}$ \\ ${ }^{a}$ Resource Economics Group, Humboldt-University zu Berlin, Unter den Linden 6, 10099 \\ Berlin, Germany \\ ${ }^{b}$ Department of Civil Engineering, Johns Hopkins University, Baltimore, USA \\ ${ }^{c}$ German Institute for Economic Research (DIW Berlin), Mohrenstr. 58, 10117 Berlin, \\ Germany
}

\begin{abstract}
Distributed photo-voltaic (PV) generation is one of the pillars of energy transitions around the world, but its deployment in the distribution grid requires costly reinforcements and expansions. Prosumage - consisting of a household-level PV unit coupled with a battery storage system - has been proposed as an effective means to facilitate the integration of renewable energy sources and reduce distribution grid stress. However, tapping its full potential requires regulatory interventions; otherwise, system costs could rise despite increasing flexibility. We analyze the effectiveness of different policy schemes to mitigate the need for distribution capacity expansion by incentivizing beneficial storage operation. Our novel top-down modeling approach allows analyzing effects on market prices, storage dispatch, induced distribution grid requirements, system costs, and distributional implications. Numerical results for German power system data indicate that required distribution grid requirements can be reduced through simple feed-in policies. A uniform limit on maximum grid feed-in can leave distribution system operators better off, even if they fully compensate prosumage households for foregone revenue. Policies imposing more differentiated limits at the regional level result in only marginal efficiency improvements. Complete self-sufficiency (autarky) is socially undesirable, as it confines important balancing potential and can increase system costs despite adding storage.
\end{abstract}

Keywords: Residential storage, renewable integration, distribution system operator, prosumage, policy, multi-level games, MPEC

JEL: C61, L94, Q41, Q42

\footnotetext{
* Corresponding author

Email address: roman.mendelevitch@hu-berlin.de (Roman Mendelevitch)
} 


\section{Introduction}

Distributed solar photo-voltaic (PV) generation is one of the pillars of the energy transition in Germany, Europe, and around the world. Its deployment at the distribution grid level poses new challenges to distribution system operators (DSOs), who manage and operate medium and low-voltage grids (Pudjianto et al. 2013). ${ }^{1}$ As regulated regional monopolies, DSOs are required to guarantee high quality and high reliability of services at all times. Thus, distribution grids are sized to handle even very rare peak events (Resch et al., 2017). Previously, dimensioning of distribution grids was driven by residential loads, where the probability of simultaneous peaks is low. By contrast, on a sunny day, all PV units in a region may generate close to their peak output simultaneously. Hence, the dimensioning of distribution grid infrastructure is now driven to an increasing degree by PV feed-in (Spiliotis et al., 2016). For Germany, studies have estimated additional investment requirements in the distribution grid of 23-49 billion EUR for the period 2015-2032 due to deployment of renewable energy sources (RES) (German Federal Ministry for Economic Affairs and Energy, 2014).

At the same time, decreasing battery storage costs (Schmidt et al., 2017) have led to the increased deployment of coupled PV battery systems (Kairies et al., 2016, Navigant Research, 2016). In Germany, such coupled systems will in many cases be more profitable than stand-alone PV installations from 2020 onwards (Dietrich and Weber, 2018). Extending the concept of electricity-

\footnotetext{
${ }^{1}$ Originally, the electrical networks were designed in a linear fashion: large-scale conventional generation units produced electricity at favorable locations and fed it into the highvoltage transmission system $(220 \mathrm{kV}$ and $380 \mathrm{kV})$ to cover long distances between generation and demand centers. On the regional and local level, the electricity was then delivered via lower-voltage distribution grids ( 1 to $110 \mathrm{kV}$ ). Both, the unidirectionality and the clear separation of tasks of these original networks is increasingly overridden has been virtually impossible to implement in energy systems with a high share of renewable energy sources. These generation units have relatively small nominal capacities and are deployed mainly at the low-voltage and medium-voltage levels (Resch et al., 2017).
} 
producing and -consuming households (prosumers), we use the term prosumage to refer to residential households with coupled PV units and battery storage (see Schill et al., 2017, Green and Staffell, 2017). Storage connected to the household's PV unit could efficiently absorb excess PV generation that cannot be handled by the grid. However, the mere availability of additional storage in the system is no panacea. In fact, system-beneficial storage operation requires appropriate market and policy designs (Ruester et al., 2014, Green and Staffell, 2017). Analyzing these involves advanced modeling setups, such that strategic interactions between players can be considered.

In this paper, we investigate policy designs that are able to incentivize system-friendly storage dispatch. We focus on their effectiveness to reduce requirements of distribution grid expansion and their cost-efficiency on the electricity system level. We employ a novel top-down modeling approach that allows us to quantify the effects of regulatory interventions on prosumage dispatch decisions and associated DSO capacity requirements as well as resulting feedback effects on market prices and system costs. To that end, we set up a two-level model that incorporates strategic decisions of DSOs as well as interactions with prosumage, generators, and the transmission level.

We establish a link between bottom-up assessments, which focus on the individual installation level (see, e.g., López Prol and Steininger, 2017, Solano et al., 2018), and system-level analyses, which do not include any representation of the prosumage (Hinz et al., 2018) or the transmission grid level (Kubli, 2018). In our setup, regionally dispersed prosumage is aggregated within one DSO region and participates in an energy-only market with nodal pricing at the level of the high-voltage transmission system. We focus on the prosumage-induced distribution grid requirements, which we model as a dedicated link between prosumage and the transmission network.

In line with the predominant practice in most European countries, we assume the shallow grid charge scheme for the recovery of the initial grid connection cost of prosumage (Hinz et al., 2018). Therefore, costs that arise from DSO link congestion are not passed on to prosumage households, and nodal prices 
fail to reflect costs induced on the distribution grid level. We take the DSOs' perspectives and allow them to incentivize prosumage to reduce network congestion. This is distinct from other approaches in the literature, which focus on prosumage and examine merits and implications of self-consumption (Green and Staffell, 2017, Yu, 2018, Solano et al., 2018). We calibrate the model to power system data for Germany for the year 2015 and add proportional storage capacities to each small-scale PV unit. We assess the effect of different policy scenarios on nodal prices, the required capacity of the DSO link, as well as on overall system costs and distributional implications between different players in the electricity system. As a benchmark, we use a first-best system configuration, which minimizes system costs. We compare it to the status quo with and without storage, as well as to two feed-in policy scenarios and an autarky case, which assumes self-sufficiency as the goal of prosumage. We provide recommendations on simple policy interventions that support system-friendly prosumage dispatch and that prevent lock-in effects.

The remainder of the paper is structured as follows: The next section situates our work within the related literature. Section 3 provides a verbal model description while Section 4 gives a mathematical description of the players' objectives and constraints. Consecutively, we introduce the six modeled scenarios in Section 5 as well as our calibration to German power system data in Section 6 . Section 7 presents the solution strategy used to obtain the numerical results, which are provided and discussed in Section 8. Section 9 concludes and elaborates on policy implications.

\section{Background and related literature}

Our contribution connects two interrelated bodies of literature. On the one hand, it is embedded in the broader literature on options for mitigating the need for distribution grid expansion due to increasing deployment of RES, which we discuss in Section 2.1. On the other hand, it is part of the more specific debate on the merits of residential storage and prosumage, and the system-level impli- 
cations of its increasing diffusion, which we detail in Section 2.2. In bridging these two bodies of literature, we contribute to the analysis of policy design for distribution grids, which we discuss in the context of the related literature in Section 2.3. Our sophisticated model design bridges the transmission system and the distribution system levels and requires advanced modeling techniques that have only recently been employed in the literature. We detail our contribution to this literature in Section 2.4.

\subsection{Options for mitigating distribution grid expansion}

An extensive body of literature focuses on the challenges that increasing shares of RES create for distribution networks, and measures that have been proposed to mitigate them (see e.g., Agricola et al., 2012, Klobasa and Mast, 2014, German Federal Ministry for Economic Affairs and Energy, 2014). Resener et al. (2018) provide an extensive review of models for investment and operational planning that aim at optimizing distribution grid capacities given increasing RES feed-in. Instead of long-term capacity expansion, for instance, short-term operations can be altered such that the available grid capacities are sufficient (Spiliotis et al., 2016, Eyer, 2009).

Georgilakis and Hatziargyriou (2015) give an overview on methods and models for distribution grid planning that incorporate distributed RES generation. Of particular interest are studies that focus on the provision of operational flexibility. Knezović et al. (2015) review different options for utilizing flexibility from electric vehicles and discuss their potential to reduce distribution grid capacity requirements. However, the paper discusses general implications for policy design in qualitative terms only, and does not provide model-based computations to support its claims. More closely related to our work, Spiliotis et al. (2016) focus on the potential of household demand response to defer grid expansion in the case of two specific distribution networks configurations but disregard effects on the electricity system level. Resch et al. (2017) present an extensive review of potential revenue streams for battery systems in Germany and discuss their ability to provide flexibility under different operation strategies. However, 
they focus on large-scale battery systems and disregard the feedback between operation strategy, market prices, and system costs.

\subsection{Flexibility provision from coupled $P V$ and battery systems}

Storage is known for its potential to mitigate network congestion (Virasjoki et al., 2016, Denholm and Sioshansi, 2009, Agricola et al., 2012) also in the distribution grid (Schill et al., 2017, Ruester et al., 2014). However, increasing available storage capacities may also increase required grid capacities (Haller et al., 2012, Neetzow et al., Resch et al., 2017). Essential for the interplay between storage and grid is the mode of operation of the storage. Prosumage storage can be charged heuristically during peak PV feed-in or as soon as the PV generation exceeds own demand (Schill et al., 2017, Moshövel et al., 2015). Another option is price-driven operation, where the dispatch decision is triggered by real-time or projected market prices. In turn, the mode depends on market characteristics such as price formation, grid tariffs, and subsidies (Ruester et al., 2014) as well as on behavioral factors such as the goal of self-sufficiency or profit maximization (Graebig et al., 2014).

Furthermore, research has been conducted on the effects of integrated smallscale storage and PV generation, i.e., prosumage. Dominguez et al. (2017) use an integrated cost minimization approach to optimize PV and storage deployment as well as operation, considering DSO-owned storage in a distribution test network. While general system effects of prosumage are discussed and modeled by Schill et al. (2017), their quantitative analysis omits impacts on the network. More specifically, Moshövel et al. (2015) show the potential to reduce network stress induced by a single prosumage household by cutting off PV peak generation with a beneficial battery-charging strategy. They do not, however, account for feedback effects of the proposed strategies to the overall system. Green and Staffell (2017) focus on the effect of maximizing prosumage self-consumption on distribution grid requirements and find that it increases capacity requirements. Whereas their work is limited to this extreme case, in the present study we assess a set of different policy options. Also focusing on self-consumption, $\mathrm{Yu}$ 
(2018) finds that prosumage puts business models of incumbent players in the French electricity system under stress.

\subsection{Policy design for distribution grids}

Along with the literature addressing the technological and economic challenges that come with the restructuring of energy systems towards RES, there is a growing body of literature examining the regulatory interventions and market design changes necessary to make the future system work efficiently (see, e.g., Ruester et al., 2014, Pérez-Arriaga et al., 2017). One strand of this literature is concerned with the efficiency of future electricity systems. MacGill and Smith (2017) provide recommendations for future policy design based on insights from past experience with prosumers' impacts on established electricity market business models in Australia. Faerber et al. (2018) detail, based on expert interviews, how distribution network charging schemes should be adapted in the transformation towards smart grids.

Smart grids could allow for efficient distribution grid pricing. Brandstätt et al. (2017) suggests a solution to the issue of non-discriminatory data availability, which is one of the central prerequisites for reaping their full potential. Highly granular locational marginal prices (LMP) could indicate the impact of a grid user's decisions on the need for expanding the network (Sotkiewicz and Vignolo, 2006) and efficiently recover investment in network capacity, at least in theory (Bohn et al., 1984, Pérez-Arriaga et al., 2017). However, such a system is not likely to emerge for regulatory, economic, or behavioral reasons (Green and Staffell, 2017). Pérez-Arriaga et al. (2017) argues that LMPs would not be an appropriate mechanism to recover distribution network costs. It would require perfect information on the household level, which raises issues of data privacy. Moreover, their implementation might induce high price differentials even within regions, which may be socially undesirable.

Another strand of literature is concerned with the distributive implications of distribution grid charging schemes. Kubli (2018) assesses the costs induced by further diffusion of prosumage in Switzerland, and analyze how different con- 
sumer groups are affected by the recovery of these costs. Similarly, Jargstorf et al. (2015) examines the effectiveness of tariffs to internalize grid costs for prosumage. However, both articles use a system dynamics approach without detailed modeling of the techno-economical interactions on the electricity market. Hinz et al. (2018) apply a detailed electricity market model of Germany to study the effect of alternative cost recovery mechanisms for distribution grids. They check for distributive justice between different regions and assess the implications of charging generators as opposed to charging consumers. While these studies focus on radical changes of the regulatory design for distribution grid, we suggest incremental policy changes that do not deviate from the shallow grid charge scheme. These could prove to be more easily implementable steps towards adapting electricity systems.

\subsection{Modeling approach}

Analyzing the strategic interactions and technical constraints on the interplay between the transmission network, the distribution grid level, prosumage, and generators requires a sophisticated modeling design. Kubli (2018) uses a system dynamics approach but accounts neither for feedback from prosumage dispatch on distribution grid requirements nor for market price effects on prosumage dispatch. Other existing detailed electricity market models either do not incorporate prosumage (Hinz et al., 2018) or lack representation of the transmission grid (Schill et al., 2017).

Our approach follows a hierarchical decision sequence, allowing the DSOs to strategically set policy parameters while anticipating associated market outcomes and dispatch decisions of prosumage and generators. To derive numerical results, this setup can be implemented as a multi-level game structure, which is being used to an increasing extent to analyze strategic interactions in energy markets. Cardell et al. (1997) analyze generator market power in the context of transmission constraints. Transmission is disregarded by Bushnell (2003), who differentiates between hydro and thermal generation technologies for strategic interaction, as well as by Schill and Kemfert (2011) and Sioshansi (2014), who 
examine the interplay between generators and storage. While all the above studies assume that the players act in a simultaneous move game, Wang et al. (2017) analyze a hierarchical setup with a strategic storage operator anticipating her own influence on the market.

All these studies consider strategic operational behavior alone. Strategic transmission investment to mitigate generator market power is additionally taken into account by Jenabi et al. (2013), Huppmann and Egerer (2015), Zerrahn and Huppmann (2017) in multi-level games. While Huppmann and Egerer (2015) consider hierarchical decisions in transmission extension (within-country and between-countries), neither of the studies that consider strategic investment considers distribution grids or storage.

In summary, to our knowledge, there is no approach in the literature to date that comprehensively analyzes interactions between multiple distribution system operators and prosumage within a transmission network and also examines the effectiveness of different regulatory schemes while taking into account hierarchical market design. Yet such a comprehensive setup is required to study an appropriate market design that ensures a system-beneficial prosumage operation. We aim to fill this research gap with the paper at hand. Our results are highly relevant to recent debates on the integration of prosumage into energy markets and the importance of regulatory design in shaping this process.

\section{Model description}

We present the first approach to incorporate network stress on the distribution grid level into a large- scale electricity DC-load flow model. Our setup consists of a multi-nodal TSO network, which connects demand centers and

large-scale generation (conventional and renewable). As we focus on the interaction of residential prosumage with the distribution grid, we distinguish between prosumage and other (non-prosumage) demand. Prosumage consists of prosumage demand, small-scale PV, and storage (Figure 1, right). At each TSO node, prosumage is connected to the TSO network via a dedicated DSO 
link. This link can be interpreted as the dedicated cumulative DSO capacity necessary to allow prosumage integration into the system. Regional DSOs ensure sufficient DSO link capacity to accommodate all prosumage inflows and outflows, while minimizing their costs. We deliberately leave out all other parts of the distribution grid, for instance, those connecting non-prosumage demand or RES to the TSO network. Technically, we do so by locating all non-prosumage demand and generation directly at the TSO node (Figure 1, left).

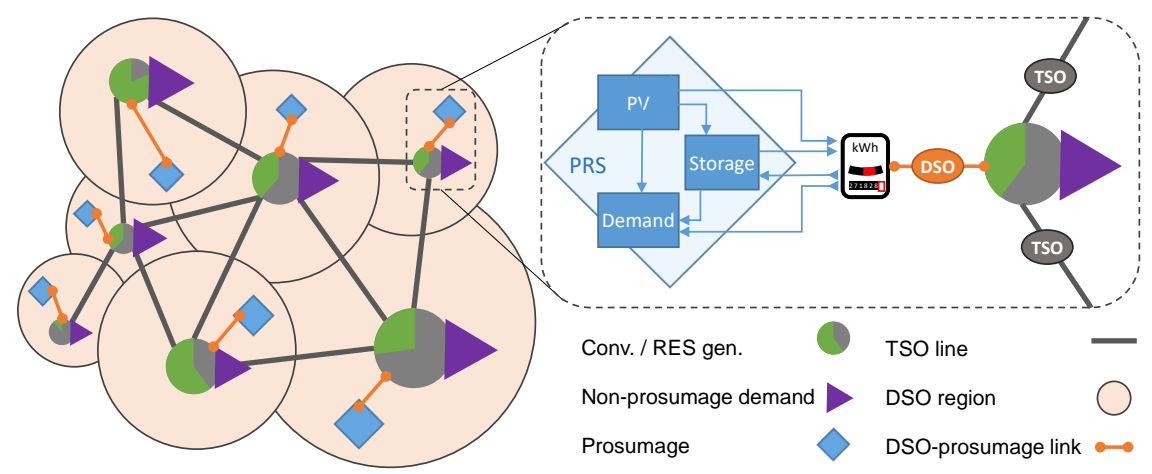

Figure 1: Left: Illustrative transmission network topology with associated regional DSOs at each TSO node. In every DSO region there exists representative non-prosumage demand, conventional and RES generation as well as prosumage, which is connected to the TSO node via a dedicated link. Right: Prosumage consists of demand, small-scale PV and storage and connects to the TSO network via a DSO link.

Generation capacities as well as demand and storage capacity are exogenous and RES feed-in is fluctuating. Any demand can be supplied from conventional generation, RES (both large-scale and prosumage-scale), storage or via import from other TSO nodes. Generators and prosumage make short term dispatch decisions, maximizing profits based on TSO nodal prices. These prices reflect possible TSO network constraints, but disregard congestion induced on the DSO level. This constitutes a market failure as prosumage does not properly internalize costs on the DSO level associated with its dispatch.

We use a set of scenarios to examine this market failure and to assess the potential of different polices to alleviate it. On the one hand, the TSO can act as a benevolent planner, minimizing total system costs and thereby achieving a 
first-best solution. On the other hand, we examine scenarios where the DSOs have the option to provide incentive payments to prosumage to alter the dispatch and reduce required distribution grid capacities. This allows us to partially internalize the grid externality and achieve a second-best solution.

This new option implies a Stackelberg game for each DSO. On the lower decision level, conventional power generators and prosumage are price-takers and Stackelberg followers in an equilibrium energy-only market. The regional DSOs can introduce incentive payments and thereby alter the equilibrium outcome. Acting as Stackelberg leaders on the upper decision level, strategic regional DSOs anticipate the equilibrium outcomes and balance incentive payments and required link capacity. Mathematically, this setup constitutes a Mathematical Program under Equilibrium Constraints (MPEC) for each of the DSOs.

The TSO grid connects the DSOs. Their decisions (optimal strategies) are therefore also not mutually independent: The chosen policy of one DSO will influence the choices of others, as a more restrictive capacity in one region and resulting higher nodal prices might incentivize an increase in prosumage feed-in in other regions. This in turn would increase the compensation required to make prosumage by local households indifferent. Enforcing an equilibrium between the DSOs would require solving an Equilibrium Program under Equilibrium Constraints (EPEC) (Ruiz et al., 2012). Solution methods for EPECs are currently limited to small-scale applications (Gabriel et al., 2012). We leave the exact solution of this problem to future research, as the purpose of the paper at hand is to provide empirically relevant results for large-scale systems. In this paper, we approximate the EPEC's solution by decoupling the respective DSO problems and instead solve a set of separate MPECs. We provide more details on the solution strategy in Section 7 and Appendix A.

\section{Mathematical model formulation}

In the following section, each player's optimization problem, consisting of the objective functions and constraints introduced in section 3 , is explained in 
full detail.

\subsection{Sets, parameters, variables}

An overview of sets, parameters and variables is given in the Tables 1 and 2 . We use lower- case letters for variables (endogenous to the model) and uppercase letters for parameters (exogenous to the model). Nodes of the TSO network are denoted by $n, m \in \mathcal{N}=\{1, \ldots, N\}$. Lines connecting the TSO nodes are denoted by $l \in \mathcal{L}=\{1, \ldots, L\}$. Time slices are denoted by $t \in \mathcal{T}=\{1, \ldots, T\}$.

\subsection{The prosumage household's problem}

There is one aggregate representative prosumage household connected to each of the TSO nodes $n$. The prosumage household's objective function is given in Eq. 1 and is the sum of the cost of purchasing electricity on the market to supply own demand $m \mathcal{2} d_{n, t}$ or to be stored in the storage $m \mathscr{2} s_{n, t}$, minus the revenue from selling PV generation $p v 2 m_{n, t}$ and electricity from storage to the market $s 2 m_{n, t}$. Each of these transactions is valued with market price $p_{n, t}^{T S O}$, which corresponds to the nodal price at the adjacent transmission network node. Demand that cannot be satisfied (lost load) $l o l_{n, t}^{P R S}$ incurs costs of the value of lost load VOLL. In addition, the prosumage household might receive compensation for an imposed policy that would restrict the prosumage operation. For our particular setup, this is depicted by the term $\left(1-\alpha_{n}\right) \cdot \bar{G}_{n}^{P R S}$. $\lambda_{n, t}^{\alpha}$. We devote Section 5 to explaining the policy-induced compensations in more detail.

$$
\begin{aligned}
\min _{\operatorname{var}^{P R S}} o b j_{n}^{P R S}=\sum_{t} p_{n, t}^{T S O} & \cdot\left(m 2 d_{n, t}+m 2 s_{n, t}-s 2 m_{n, t}-p v 2 m_{n, t}\right) \\
& +\operatorname{lol}_{n, t}^{P R S} \cdot \operatorname{VOLL}-\left(1-\alpha_{n}\right) \cdot \bar{G}_{n}^{P R S} \cdot \lambda_{n, t}^{\alpha}
\end{aligned}
$$

The prosumage household is subject to several constraints (Eqs. 2-9). The households own demand $D_{n, t}^{P R S}$ can be satisfied by three sources: its own supply from PV generation $p v 2 d_{n, t}$, from storage $s 2 d_{n, t}$, or from the market $m \mathscr{2} d_{n, t}$. Under some circumstances, these might not be sufficient to satisfy demand, 
Table 1: Sets and parameters used in the model.

\begin{tabular}{|c|c|}
\hline Name & Description \\
\hline \multicolumn{2}{|l|}{ Sets } \\
\hline$n, m \in \mathcal{N}=\{1, \ldots, N\}$ & TSO nodes \\
\hline$l \in \mathcal{L}=\{1, \ldots, L\}$ & TSO lines \\
\hline$\tau \in \mathcal{D}=\{1, \ldots, 365\}$ & Days of the year \\
\hline$t \in \mathcal{T}=\{1, \ldots, 8760\}$ & Hours of the year \\
\hline$t \in \mathcal{T}_{\tau}$ & Hours of day $\tau$ \\
\hline \multicolumn{2}{|l|}{ Parameters } \\
\hline$D_{n, t}^{P R S}$ & Demand from prosumage $[\mathrm{GW}]$ \\
\hline$G_{n, t}^{P R S}$ & PV generation from prosumage $[\mathrm{GW}]$ \\
\hline $\bar{G}_{n}^{P R S}$ & Prosumage PV capacity $[\mathrm{GW}]$ \\
\hline $\bar{E}_{n}^{P R S}$ & Energy capacity of storage [GWh] \\
\hline $\bar{P}_{n}^{P R S}$ & Storage power capacity $[\mathrm{GW}]$ \\
\hline$E_{n}^{P R S}$ & Inital storage level $[\mathrm{GWh}]$ \\
\hline$\eta$ & Round-trip storage efficiency [-] \\
\hline$C_{n, t}^{G E N}$ & Generation cost parameter $[(\mathrm{EUR} / \mathrm{MWh}) / \mathrm{GW}]$ \\
\hline $\bar{G}_{n, t}^{G E N}$ & Seasonally available generation capacity $[\mathrm{GW}]$ \\
\hline$M C^{D S O}$ & DSO unit capacity cost per hour of grid use $[$ TEUR/(GW·h $)]$ \\
\hline$D_{n, t}$ & Non-prosumage demand [GW] \\
\hline$G_{n, t}^{R E S}$ & Non-prosumage generation potential from RES [GW] \\
\hline$R_{n, t}$ & Residual demand of non-prosumage demand and RES [GW] \\
\hline$V O L L$ & Value of lost load [EUR/MWh] \\
\hline$H_{l, n}$ & Network transfer matrix $[1 / \Omega]$ \\
\hline$B_{n, m}$ & Network susceptance matrix $[1 / \Omega]$ \\
\hline $\bar{F}_{l}^{T S O}$ & Capacities of TSO lines [GW] \\
\hline
\end{tabular}


Table 2: Primal variables (var) and dual variables $(d u)$ of the model.

\begin{tabular}{|c|c|c|}
\hline Superset & Name & Description \\
\hline \multirow{11}{*}{$\operatorname{var}^{P R S}$} & Variables & \\
\hline & $p v 2 d_{n, t}$ & Flow from prosumage PV to prosumage demand $[\mathrm{GW}]$ \\
\hline & $s 2 d_{n, t}$ & Flow from prosumage storage to prosumage demand (self-consumption) $[\mathrm{GW}]$ \\
\hline & $m 2 d_{n, t}$ & Flow from market to prosumage demand (purchase) $[\mathrm{GW}]$ \\
\hline & $m 2 s_{n, t}$ & Flow from market to prosumage storage (purchase) $[\mathrm{GW}]$ \\
\hline & $s 2 m_{n, t}$ & Flow from prosumage storage to market (sale) $[\mathrm{GW}]$ \\
\hline & $p v 2 s_{n, t}$ & Flow from prosumage PV to prosumage storage $[\mathrm{GW}]$ \\
\hline & $p v 2 m_{n, t}$ & Flow from prosumage $\mathrm{PV}$ to market (sale) $[\mathrm{GW}]$ \\
\hline & $e_{n, t}^{P R S}$ & Energy level of storage [GWh] \\
\hline & $l o l_{n, t}^{P R S}$ & Lost load at prosumage \\
\hline & $\operatorname{curt}_{n, t}^{P R S}$ & Curtailment at prosumage \\
\hline $\operatorname{var}^{G E N}$ & $g_{n, t}^{G E N}$ & Conventional generation $[\mathrm{GW}]$ \\
\hline \multirow[t]{2}{*}{$\operatorname{var}^{D S O}$} & $\bar{f}_{n}^{D S O}$ & DSO capacity connected to node $n[\mathrm{GW}]$ \\
\hline & $\alpha_{n}$ & Policy variable $[-]$ \\
\hline \multirow[t]{5}{*}{$\operatorname{var}^{T S O}$} & $f_{l, t}^{T S O}$ & Flow at TSO line $[\mathrm{GW}]$ \\
\hline & $i m_{n, t}^{T S O}$ & Inflow from TSO grid $[\mathrm{GW}]$ \\
\hline & $l o l_{n, t}^{T S O}$ & Lost load at TSO node $[\mathrm{GW}]$ \\
\hline & $\operatorname{curt}_{n, t}^{T S O}$ & Curtailment at TSO node $[\mathrm{GW}]$ \\
\hline & $\theta_{n, t}$ & Phase angle $[\mathrm{deg}]$ \\
\hline \multicolumn{3}{|l|}{ Duals } \\
\hline \multirow[t]{8}{*}{$d u^{P R S}$} & $\lambda_{n, t}^{P V}$ & Shadow price on PV generated electricity [EUR/MWh] \\
\hline & $\lambda_{n, t}^{D}$ & Shadow price on electricity consumed by prosumage [EUR/MWh] \\
\hline & $\lambda_{n, t}^{S T O R}$ & Shadow price on electricity in the storage [EUR/MWh] \\
\hline & $\lambda_{n, t}^{C H A R G E}$ & Shadow price on storage charge $[\mathrm{EUR} / \mathrm{MW}]$ \\
\hline & $\lambda_{n, t}^{D I S C H}$ & Shadow price on storage discharge $[\mathrm{EUR} / \mathrm{MW}]$ \\
\hline & $\lambda_{n, t}^{\bar{E}}$ & Shadow price on storage capacity [EUR/MWh] \\
\hline & $\lambda_{n}^{E}$ & Shadow price for refilling the storage $[\mathrm{EUR} / \mathrm{MWh}]$ \\
\hline & $\lambda_{n, t}^{\alpha}$ & Shadow price on policy constraint $[\mathrm{EUR} / \mathrm{MW}]$ \\
\hline$d u^{G E N}$ & $\lambda_{n, t}^{\bar{G}}$ & Shadow price on generation capacity [EUR/MW] \\
\hline$d u^{T S O}$ & $p_{n, t}^{T S O}$ & Wholesale electricity price at the TSO node [EUR/MWh] \\
\hline
\end{tabular}


resulting in lost load $l o l_{n, t}^{P R S}$ (Eq. 2). For each point in time $t, \mathrm{PV}$ feed-in $G_{n, t}^{P R S}$ can be balanced in four ways: self-consumption $p v 2 d_{n, t}$, sales to the market $p v 2 m_{n, t}$, storage $p v 2 s_{n, t}$, or as a measure of last resort, curtailment $\operatorname{curt}_{n, t}^{P R S}$ (Eq. 3). Eq. 4 gives the temporal balance for the storage, where the current energy level $e_{n, t}^{P R S}$ equals the previous level reduced by outflows and increased by inflows. The latter are reduced by the round-trip efficiency $\eta$. The storage level cannot exceed the energy storage capacity $\bar{E}_{n}^{P R S}$ (Eq. 5). Moreover, in(out)-flow into storage cannot exceed its power capacity $\bar{P}_{n}^{P R S}$ (Eqs. 6 and 7). Eq. 8 sets the boundary conditions for storage, where final $(t=T)$ storage levels $e_{n t, T}^{P R S}$ have to be equal to initial storage levels $E_{n}^{P R S}$. The final Eq. 9 depicts the operating constraints that arise from the institutional design. The prosumage feed-in to the market cannot be higher than a fraction $\alpha_{n}$ of its own PV generation capacity $\bar{G}_{n}^{P R S}$ (also see Figure 2). We devote Section 5 to analyzing the respective policy options in more detail.

$$
\begin{aligned}
0= & p v 2 d_{n, t}+s 2 d_{n, t}+m 2 d_{n, t}+l o l_{n, t}^{P R S}-D_{n, t}^{P R S}\left(\lambda_{n, t}^{D}\right) \\
0= & -p v 2 d_{n, t}-p v 2 s_{n, t}-p v 2 m_{n, t}-c u r t_{n, t}^{P R S}+G_{n, t}^{P R S} \quad\left(\lambda_{n, t}^{P V}\right) \\
0= & \left\{\begin{array}{l}
-s 2 d_{n, t}+\eta \cdot m 2 s_{n, t}-s 2 m_{n, t}+\eta \cdot p v 2 s_{n, t}-e_{n, t}^{P R S}+E_{n}^{P R S}, \text { if } t=1 \\
-s 2 d_{n, t}+\eta \cdot m 2 s_{n, t}-s 2 m_{n, t}+\eta \cdot p v 2 s_{n, t}-e_{n, t}^{P R S}+e_{n, t-1}^{P R S}, \text { otherwise }
\end{array}\right. \\
& \left(\lambda_{n, t}^{S T O R}\right) \\
0 \leq & e_{n, t}^{P R S}-\bar{E}_{n}^{P R S} \quad\left(\lambda_{n, t}^{E}\right) \\
0 \leq & m 2 s_{n, t}+p v 2 s_{n, t}-\bar{P}_{n}^{P R S} \\
0 \leq & s 2 d_{n, t}+s 2 m_{n, t}-\bar{P}_{n}^{P R S} \quad\left(\lambda_{n, t}^{C H A R G E}\right) \\
0= & e_{n t, T}^{P R S}-E_{n}^{P R S} \quad\left(\lambda_{n, t}^{D I S C H}\right) \\
0 \leq & \alpha_{n} \cdot \bar{G}_{n}^{P R S}-s 2 m_{n, t}-p v 2 m_{n, t} \quad\left(\lambda_{n, t}^{\alpha}\right)
\end{aligned}
$$

\subsection{The generator's problem}

Besides the prosumage household, there is one representative (non-strategic) operator of conventional generation at each TSO node. The operator maximizes 
its revenue by dispatching conventional generation $g_{n, t}$ to provide electricity. We assume a quadratic generation cost function $g_{n, t}^{2} / 2 \cdot C_{n, t}^{G E N}$ characterized by the cost parameter $C_{n, t}^{G E N} .^{2}$ The generated electricity is sold at market price $p_{n, t}^{T S O}$ (Eq. 10). Furthermore, the generator is constrained by the available generation capacity $\bar{G}_{n, t}^{G E N}$ (Eq. 11).

$$
\begin{aligned}
& \min _{v_{\operatorname{rar}}^{G E N}} o b j_{n}^{G E N}=g_{n, t}^{2} / 2 \cdot C_{n, t}^{G E N}-g_{n, t} \cdot p_{n, t}^{T S O} \\
& \text { s.t. } \quad 0 \leq g_{n, t}-\bar{G}_{n, t}^{G E N} \quad\left(\lambda_{n, t}^{\bar{G}}\right)
\end{aligned}
$$

\subsection{The DSO's problem}

The DSO is in charge of the link that connects the prosumage household to the TSO node. ${ }^{3}$ The objective of the DSOs is to minimize capacity costs of the DSO link as well as compensation costs paid to incentivize the prosumage household to reduce its network use. To account for the length of the capacity planning horizon, marginal capacity investment costs $M C^{D S O}$ are multiplied by the number of hours considered, i.e., the cardinality of $t(|\mathcal{T}|)$ (Eq. 12).

$$
\min _{\operatorname{var}^{D S O}} o b j_{n}^{D S O}=\bar{f}_{n}^{D S O} \cdot M C^{D S O} \cdot|\mathcal{T}|+\sum_{t}\left(1-\alpha_{n}\right) \cdot \bar{G}_{n}^{P R S} \cdot \lambda_{n, t}^{\alpha}
$$

This is subject to two balance constraints: one for the inflow, i.e., from the TSO node to the prosumage household (Eq. 13), and one for outflows, i.e., from the prosumage household to the TSO node (Eq. 14). Eq. 13 is straightforward, ensuring that DSO link capacity is large enough for the inflow. Eq. 14 is

\footnotetext{
${ }^{2}$ The parameter $C_{n, t}^{G E N}$ thus describes a linear marginal cost function of the form $M C_{n, t}^{G E N}\left(g_{n, t}\right)=C_{n, t}^{G E N} \cdot g_{n, t}$.

${ }^{3}$ In reality, the DSO is responsible for supplying grid connectivity for all types of consumers, not only prosumage households, but also regular households, industrial operations, as well as utility-scale renewable generation. In this paper, we focus on the interaction among prosumage households, DSO, and TSO. Therefore, we aggregate residual demand $R_{n, t}$ (excluding prosumage) to the TSO node level.
} 
connected to the policy design and obtains its effectiveness in connection with Eq. 9. It guarantees a minimum share of $\alpha_{n}$ of the maximum PV generation capacity for prosumage $\left(\bar{G}_{n}^{P R S}\right)$ as admissible outflow (also see Figure 2).

$$
\begin{aligned}
& 0 \leq \bar{f}_{n}^{D S O}-m 2 d_{n, t}-m 2 s_{n, t} \\
& 0 \leq \bar{f}_{n}^{D S O}-\alpha_{n} \cdot \bar{G}_{n}^{P R S}
\end{aligned}
$$

\subsection{The TSO's problem}

Finally, the TSO takes the system perspective by trying to minimize total system costs comprised of generation costs, costs of lost loads, and necessary investments in the DSO networks (Eq. 15). ${ }^{4}$

$$
\begin{aligned}
\min _{\operatorname{var}^{T S O}} o b j^{T S O}= & \sum_{n, t}\left(1 / 2 \cdot g_{n, t}^{2} \cdot C_{n, t}^{G E N}+\left(\operatorname{lol}_{n, t}^{P R S}+\operatorname{lol}_{n, t}^{T S O}\right) \cdot V O L L\right) \\
& +\sum_{n}\left(\bar{f}_{n}^{D S O} \cdot M C^{D S O} \cdot|\mathcal{T}|\right)
\end{aligned}
$$

Furthermore, it ensures balancing of the overall system flows, which follow the usual linearized DC-load flow approach of Kirchhoff's Laws (see, e.g., Schweppe et al., 1988). The nodal balance is given in Eq. 16. Here the residual nonprosumage demand $R_{n, t}$ is given from the non-prosumage demand $D_{n, t}$ and potential generation from RES $G_{n, t}^{R E S}$, i.e., $R_{n, t}=D_{n, t}-G_{n, t}^{R E S}$. As in the prosumage household's problem, we allow for curtailment curt $_{n, t}^{T S O}$ and lost load $l o l_{n, t}^{T S O}$ in case there is an excess or a shortage in power. Nodal net imports via the TSO grid are represented by $i m_{n, t}^{T S O}$. Imports are calculated using the network susceptance matrix $B_{n, m}$ and nodal phase angle difference $\theta_{n, t}$ to a swing node $\hat{n}$ (Eq. 17 and 21). The net flow on a TSO line is denoted by $f_{l, t}^{T S O}$ and computed from the phase angle and the network transfer matrix $H_{l, n}$ (Eq. 18). TSO line capacity $\bar{F}_{l}^{T S O}$ has to accommodate positive and negative TSO network flows (Eqs. 19 and 20).

\footnotetext{
${ }^{4}$ For now, we abstract from investment into the TSO grid.
} 


$$
\begin{aligned}
0= & -i m_{n, t}^{T S O}+m 2 d_{n, t}+m 2 s_{n, t}-s 2 m_{n, t}-p v 2 m_{n, t}-g_{n, t}+R_{n, t} \\
& +\operatorname{curt}_{n, t}^{T S O}-\operatorname{lol} l_{n, t}^{T S O}\left(p_{n, t}^{T S O}\right) \\
0= & -i m_{n, t}^{T S O}+B_{n, m} \cdot \theta_{n, t} \\
0= & -f_{l, t}^{T S O}+H_{l, n} \cdot \theta_{n, t} \\
0 \leq & -f_{l, t}^{T S O}+\bar{F}_{l} \\
0 \leq & f_{l, t}^{T S O}+\bar{F}_{l} \\
0= & \theta_{\hat{n}, t}
\end{aligned}
$$

\section{Scenarios}

In this section, we introduce six scenarios to evaluate different policy mechanisms. They differ in their specific parametrizations, in variations of the players' objectives as well as through the existence or non-existence of strategic interactions between them. The scenarios entitled No storage, Smart, Autarky, No policy, KfW policy and DSO-wise policy are described below and a summary is provided in Table 3. While No storage, Smart and No policy can be implemented as single-level optimization problems, Autarky, KfW policy and DSOwise policy incorporate strategic interactions between players in a Stackelberg leader-follower setting, and thus require a more sophisticated solution approach.

In the No storage scenario, the TSO - a benevolent system planner - has perfect information and comprehensive decision-making power to minimize total system costs. It takes into account costs of conventional generation and necessary distribution grid investments. Yet, in contrast to all other scenarios, there is no prosumage storage capacity available in the system. The second scenario, Smart, envisions a smart system setup, where storage systems are available in prosumage households and can be utilized by the TSO to further minimize sys-

tem costs. In the No policy scenario, DSO capacity and respective costs are excluded from the TSOs consideration. This scenario is representative of a situation in which dispatch decisions on the prosumage level are driven by market 
Table 3: Overview of scenarios by storage availability, game structure, maximum feed-in as well consideration of DSO link costs. While in No storage and Smart, prosumage feed-in is optimized from a total system cost perspective, it is unrestricted in No policy, set to a generic limit in $K f W$ policy, and is set to a DSO-specific limit in DSO-wise policy. No prosumage feed-in is allowed for the Autarky scenario.

\begin{tabular}{|c|c|c|c|c|c|}
\hline Scenario & Storage & $\begin{array}{c}\text { Max. prosum- } \\
\text { age feed-in }\end{array}$ & $\begin{array}{r}\text { Compen- } \\
\text { sation }^{\mathbf{a}}\end{array}$ & Costs of DSO link & Game structure \\
\hline No storage & & optimized & implicit & fully internalized & min system cost \\
\hline Smart & $\checkmark$ & optimized & implicit & fully internalized & min system cost \\
\hline Autarky & $\checkmark$ & no feed-in & $\mathrm{n} / \mathrm{a}$ & partly avoided & Stackelberg game \\
\hline No policy & $\checkmark$ & unrestricted & $\mathrm{n} / \mathrm{a}$ & disregarded & min system cost \\
\hline$K f W$ policy & $\checkmark$ & generic limit & explicit & partly internalized & Stackelberg game \\
\hline DSO-wise policy & $\checkmark$ & DSO-specific limit & explicit & partly internalized & Stackelberg game \\
\hline
\end{tabular}

${ }^{a}$ Compensation for prosumage dispatch restrictions by DSO

prices and prosumage does not take into account its effect on DSO capacity requirements.

In addition to the three single-level, cost-minimization scenarios, we evaluate three multi-level scenarios with strategic interactions between prosumage and DSOs. Interaction is reflected in the compensation terms of their objective functions as well as Eq. 9, which imposes an operational constraint on prosumage, reducing the allowable feed-in to the market (Figure 2). We deploy a straightforward mechanism characterized by the choice of $\alpha_{n}$. An adequate choice of $\alpha_{n}$ can reduce the market failure induced by the prosumage households ignorance of the DSO capacity costs. If $\alpha_{n}$ is large there are no or few restrictions on the prosumage grid feed-in, while for $\alpha_{n}=0$ no feed-in is allowed.

In the Autarky scenario, prosumage is determined to maximize self-sufficiency, i.e., the share of own PV generation in its demand (see Luthander et al., 2015), and to reduce market interactions. This can be represented by $\forall n: \alpha_{n}=0$. As a consequence, prosumage will never feed into the DSO link and will instead use storage to satisfy its own demand from its own generation. Yet, if demand 

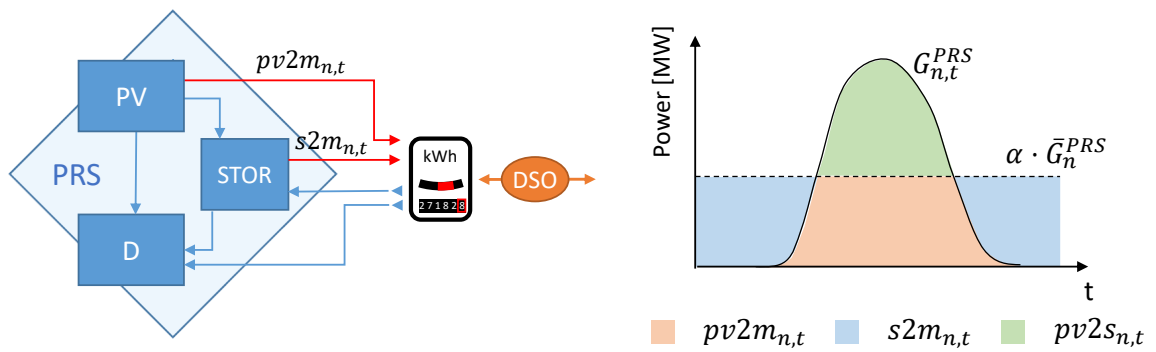

Figure 2: Possible prosumage feed-in after introduction of the policy variable $\alpha_{n}$. Maximum grid feed-in from the prosumage into the DSO link $\left(p v 2 m_{n, t}+s 2 m_{n, t}\right)$ is reduced to a fraction $\alpha_{n}$ of the prosumage PV capacity. Excess PV generation must be consumed, stored or curtailed.

cannot be met by either PV generation or storage, prosumage can still purchase electricity from the market using the DSO link. Even though the Autarky scenario imposes operational costs on prosumage, we abstain from compensation payments as we assume that the autarky decision is made by the prosumage household for non-monetary reasons (Graebig et al., 2014).

In the KfW policy scenario, the policy is exogenously set for all DSOs such that $\forall n: \alpha_{n}=0.5$. The name is derived by a storage promotion program that is in place in Germany and supported by the state-backed investment bank KfW. ${ }^{5}$ As a consequence, prosumage feed-in is limited to half the available prosumage PV capacity. This in turn reduces the required distribution grid capacity and therefore grid investment costs. Yet, the restriction of the prosumage households dispatch decisions might reduce revenues. The shadow price $\lambda_{n, t}^{\alpha}$ of Eq. 9 provides a unit of measurement for the foregone marginal revenue due to the imposed restriction. To compensate the prosumage household for this loss, we include the compensation payment $\sum_{t}\left(1-\alpha_{n}\right) \cdot \bar{G}_{n}^{P R S} \cdot \lambda_{n, t}^{\alpha}$ from the DSO to the prosumage household. As the desired grid feed-in from the prosumage household

\footnotetext{
${ }^{5}$ In the German storage support scheme, favorable credit terms for household storage units are granted if the maximum share of PV feed-in is limited to $50 \%$ of PV generation capacity through storage operation (KfW, 2016).
} 
is unknown to the DSO, it compensates up to the maximum potential feed-in inhibited by the policy $\left(1-\alpha_{n}\right) \cdot \bar{G}_{n}^{P R S}$, making the prosumage household at least indifferent compared to a scenario without a policy.

In the $D S O$-wise policy scenario, we assume that $\alpha_{n}$ can be chosen freely by the $n$th DSO, i.e., every DSO restricts the dispatch of its associated prosumage. Again, we assume that the respective DSO has to compensate the prosumage household. The compensation scheme is the same as under the KfW policy. Yet, the DSO-specific choice allows each DSO to balance costs for capacity investment and prosumage compensation. The compensation increases the more restrictive the policy gets: on the one hand directly from a decreasing $\alpha_{n}$, and on the other hand indirectly from the increase of shadow price $\lambda_{n, t}^{\alpha}$, which is a function of the market price $p_{n, t}^{T S O}$.

\section{Model calibration}

We calibrate the model with state-wise (Bundesland) aggregated data on the German electricity system (i.e., $n \in \mathcal{N}=\{\mathrm{BB}, \mathrm{BE}, \mathrm{BW}, \mathrm{BY}, \mathrm{HB}, \mathrm{HE}$, HH, MV, NI, NRW, RP, SH, SL, SN, ST, TH $\}),{ }^{6}$ for the year 2015. Hence, we assume that the distribution grid of each respective state is operated by one DSO. ${ }^{7}$ Wherever possible, we use the electricity data provided in Kunz et al. (2017). The relevant available data on conventional plants, renewable energy capacities $^{8}$, time series for wind feed-in, demand, as well as properties of the transmission grid are all aggregated to a state level. Furthermore, for each state we estimate a linear approximation to its unit level merit order curve using a

\footnotetext{
${ }^{6}$ German Federal States: Brandenburg (BB), Berlin (BE), Baden-Wurttemberg (BW), Bavaria (BY), Hesse (HE), Bremen (HB), Hamburg (HH), Mecklenburg-West Pomerania (MV), Lower Saxony (NI), North Rhine-Westphalia (NRW), Rhineland-Palatinate (RP), Schleswig-Holstein (SH), Saarland (SL), Saxony (SN), Saxony-Anhalt (ST), Thuringia (TH)

${ }^{7}$ Even though there are about 890 different DSOs in Germany (BNetzA, 2018), we reduce complexity while maintaining DSO diversity by using this assumption.

${ }^{8}$ We consider the renewable technologies run off rivers, biomass, geothermal, hydro, and waste as non-dispatchable units with partly seasonal availabilities.
} 
least-squares fit. The derived marginal generation costs are corrected for the availability of generation capacities and therefore time-dependent. The value of lost load VOLL is assumed to be $200 \mathrm{EUR} / \mathrm{MWh}$ We allocate all PV-systems $\leq 10 \mathrm{kWh}$ which amount for $5.8 \mathrm{GW}$ (Open Power System Data, 2018) ${ }^{9}$ to the prosumage. For the purpose of our analysis, we assume that each PV system is accompanied by proportional storage capacities (in total 2.9 GW, 11.6 GWh) with a round trip efficiency of 0.9 .

State-level, capacity-normalized time series for PV feed-in are taken from Koch et al. $(2016)^{10}$. We approximate the share of household consumption in load by BDEW household standard load profiles (Bundesverband der Energieund Wasserwirtschaft e.V., 2015) and total German household consumption in 2015 (Umweltbundesamt, 2017). Finally, the prosumage demand is approximated by the state-wise number of PV-systems $\leq 10 \mathrm{kWh}$ (Open Power System Data, 2018) and by assuming the average yearly demand of a single prosumage household to be $5 \mathrm{MWh}$, which is in line with Beck et al. (2016), Bertsch et al. (2017). DSO unit investment costs are assumed to be 2 EUR per MW and hour. ${ }^{11}$ An overview of parameters is given in Figure 3 and Table $4 .{ }^{12}$

We compute a whole year with an hourly temporal resolution. However, for computational reasons, we do not compute all hours of the year at once but solve all days separately. To make all days $\tau \in\{1, \ldots, 365\}$ independent of one

\footnotetext{
${ }^{9}$ We derive the share of PV-systems $\leq 10 \mathrm{kWh}$ from (Open Power System Data, 2018) but use the data on total capacity from Kunz et al. (2017).

${ }^{10}$ The study uses the weather year 2011 and the predicted distribution of PV-systems in 2020 .

${ }^{11}$ This figure is based on calculations from Klobasa and Mast (2014). The study reports 1.4 bn EUR of additional annual investment requirements for distribution grid expansions in order to integrate a capacity of $92.1 \mathrm{GW}$ in PV and wind generation for the period up to 2020. In the future, they assume these requirements will increase further by about $50 \%$ up to 2030. Distributing costs over examined hours gives us a value of $\approx 2$ EUR per MW of installed RES capacity (or potential RES feed-in) and hour.

${ }^{12} \mathrm{~A}$ comprehensive dataset can be found: Comprehensive data will be publicly provided with the final publication of the paper.
} 

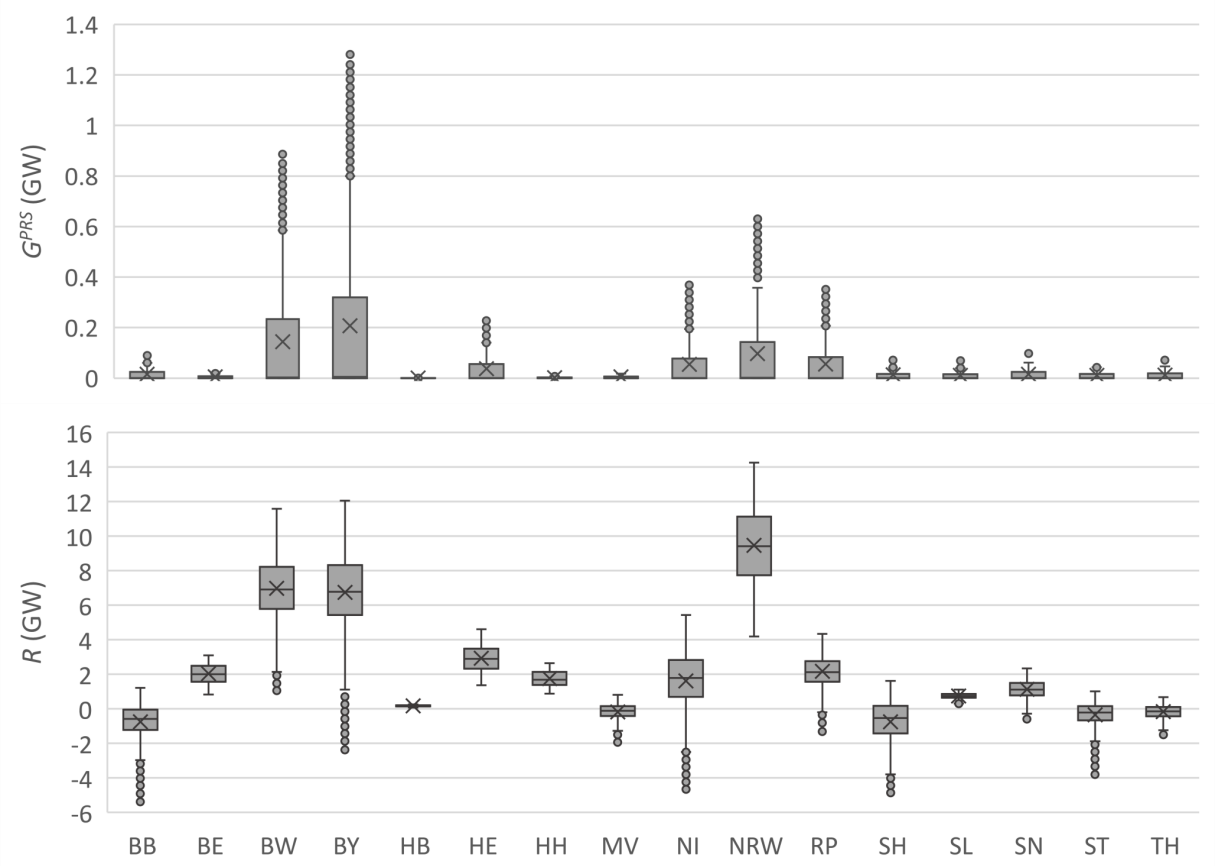

Figure 3: Overview on data by state. Top: prosumage household PV generation. Bottom: residual demand (excluding prosumage). Level and distribution of residual demand varies significantly between states. Prosumage households are concentrated on only few states. Data source: own computations on the basis of Open Power System Data (2018), Kunz et al. (2017), Koch et al. (2016). 
Table 4: Generation cost parameters as well as conventional and prosumage capacities by state. Data source: own computations on the basis of Open Power System Data (2018), Kunz et al. (2017).

\begin{tabular}{|c|c|c|c|c|c|c|c|c|c|}
\hline & \multicolumn{3}{|c|}{$C_{n, t}^{G E N}[(\mathrm{EUR} / \mathrm{MW}) / \mathrm{GW}]$} & \multicolumn{3}{|c|}{$\bar{G}_{n, t}^{G E N}[\mathrm{GW}]$} & \multirow{2}{*}{$\begin{array}{l}\bar{G}_{n}^{P R S} \\
{[\mathrm{MW}]}\end{array}$} & \multirow{2}{*}{$\begin{array}{l}\bar{P}_{n}^{P R S} \\
{[\mathrm{MW}]}\end{array}$} & \multirow{2}{*}{$\begin{array}{l}\bar{E}_{n}^{P R S} \\
{[\mathrm{MWh}]}\end{array}$} \\
\hline & Mean & Min & Max & Mean & Min & $\operatorname{Max}$ & & & \\
\hline $\mathrm{BB}$ & 20.6 & 18.5 & 24.4 & 3.1 & 2.6 & 3.4 & 135.2 & 67.6 & 270.4 \\
\hline $\mathrm{BE}$ & 50.9 & 47.6 & 54.6 & 2.1 & 1.9 & 2.2 & 46.6 & 23.3 & 93.1 \\
\hline BW & 10.5 & 9.7 & 12.1 & 6.7 & 5.7 & 7.2 & 1129.2 & 564.6 & 2258.4 \\
\hline BY & 7.9 & 7.2 & 9.4 & 9.8 & 8.2 & 10.7 & 1657.3 & 828.7 & 3314.6 \\
\hline HB & 335.7 & 314.5 & 356.9 & 0.1 & 0.1 & 0.1 & 1.7 & 0.9 & 3.4 \\
\hline $\mathrm{HE}$ & 35.1 & 32.9 & 37.3 & 2.1 & 2 & 2.3 & 323.2 & 161.6 & 646.5 \\
\hline HH & 33.6 & 31.5 & 35.7 & 1.5 & 1.4 & 1.6 & 21.7 & 10.8 & 43.4 \\
\hline MV & 87.2 & 81.7 & 92.7 & 0.7 & 0.6 & 0.7 & 44.1 & 22.1 & 88.3 \\
\hline NI & 8.8 & 8.1 & 9.9 & 8.3 & 7.4 & 9 & 514.9 & 257.5 & 1029.9 \\
\hline NRW & 2.8 & 2.6 & 3.1 & 23.5 & 21 & 25.5 & 861.8 & 430.9 & 1723.6 \\
\hline $\mathrm{RP}$ & 19.5 & 18.2 & 20.7 & 3.4 & 3.2 & 3.6 & 473.3 & 236.6 & 946.5 \\
\hline $\mathrm{SH}$ & 40.9 & 37.1 & 49.1 & 2.3 & 1.9 & 2.5 & 121.9 & 61 & 243.8 \\
\hline SL & 28.4 & 26.7 & 30.1 & 1.9 & 1.8 & 2 & 96.3 & 48.2 & 192.6 \\
\hline $\mathrm{SN}$ & 6.4 & 5.6 & 7.7 & 5.2 & 4.3 & 5.8 & 133.1 & 66.5 & 266.2 \\
\hline $\mathrm{ST}$ & 52.8 & 48 & 60.4 & 1.6 & 1.4 & 1.7 & 94.4 & 47.2 & 188.9 \\
\hline TH & 199.5 & 187.2 & 211.7 & 0.4 & 0.4 & 0.4 & 98.5 & 49.2 & 197 \\
\hline Total & & & & 72.7 & 63.9 & 78.7 & 5753 & 2876 & 11506 \\
\hline
\end{tabular}


another ${ }^{13}$, we fix the energy levels of all storage capacities at the end of every day to their initial energy levels (see Eq. 8) and assume $E_{n}^{P R S}=\bar{E}_{n}^{P R S} / 2$.

\section{Solution strategy}

The following section details the individual reformulations and steps that are necessary to solve the mathematical problems defined by the different scenarios. In section 7.1, we focus on the scenarios Smart, No storage and No policy, which are solved as single-level cost minimizations for each day of the year individually. Section 7.2 describes how we decouple the respective DSO problems from each other and solve the scenarios Autarky, KfW policy and DSO-wise policy as separate MPECs for each DSO region, again individually for each day. Finally, to derive system-level results for the whole year, we recompute all scenarios using their maximum realized daily DSO capacity as minimum capacity for the whole year, in Section 7.3.

7.1. Compute Smart, No storage and No policy scenarios as cost minimization to obtain daily necessary DSO capacity

We solve scenarios Smart, No storage and No policy as system cost minimization problems for each day, separately, without considering any strategic interactions between the players. To derive results for the smart scenario, we adapt a benevolent planner perspective for the TSO such that its objective sums all existing system costs (this is equivalent to the sum of all players' objectives if we include non-prosumage demand, renewable generators, and traders in the TSO network). Thus, we can extend the TSO problem to include the constraints of the other playersminimizing over all players' variables:

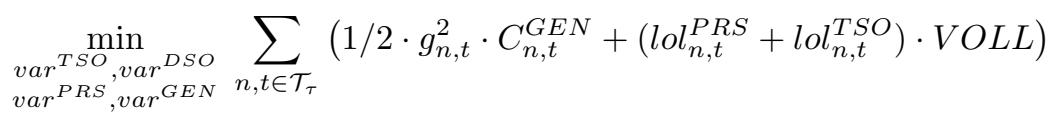

\footnotetext{
${ }^{13}$ For computation, we define a subset $\mathcal{T}_{\tau}$ for each day of the year such that $\mathcal{T}_{\tau}=\{\tau \cdot 24-$ $23, \tau \cdot 24-22, \ldots, \tau \cdot 24-0\}$ with $\tau \in\{1, \ldots, 365\}$.
} 


$$
\begin{aligned}
& +\sum_{n}\left(\bar{f}_{n, \tau}^{D S O} \cdot M C^{D S O} \cdot\left|\mathcal{T}_{\tau}\right|\right), \forall \tau \\
& \text { s.t. }(2-9),(11),(13-14),(16-20) \mid t \in \mathcal{T}_{\tau} .
\end{aligned}
$$

The problem features a convex objective function and linear constraints. Thus, the solution to the problem yields the globally optimal result (Boyd and Vandenberghe, 2004).

To implement the No storage scenario, we additionally parametrize

$$
\bar{E}_{n}^{P R S}=\bar{P}_{n}^{P R S}=0,
$$

such that no storage capacity is available.

Finally, the No policy scenario neglects DSO capacity constraints and associated costs in its objective. We can write the associated problem for each day as

$$
\min _{\substack{\operatorname{var}^{T S O} \\ \operatorname{var}^{P R S}, \operatorname{var}^{G E N}}} \sum_{n, t \in \mathcal{T}_{\tau}}\left(1 / 2 \cdot g_{n, t}^{2} \cdot C_{n, t}^{G E N}+\left(\operatorname{lol}_{n, t}^{P R S}+l o l_{n, t}^{T S O}\right) \cdot V O L L\right), \forall \tau
$$

$$
\text { s.t. }(2-9),(11),(16-20) \mid t \in \mathcal{T}_{\tau} \text {. }
$$

We compute the required DSO capacity ex post from the maximum flow that occurs on the DSO link.

$$
\bar{f}_{n, \tau}^{D S O}=\max \left(\max _{t \in \mathcal{T}_{\tau}}\left(p v \mathcal{2} m_{n, t}+s \mathscr{2} m_{n, t}\right), \max _{t \in \mathcal{T}_{\tau}}\left(m \mathscr{2} d_{n, t}+m \mathscr{2} s_{n, t}\right)\right)
$$

7.2. Compute Autarky, KfW policy and DSO-wise policy as two-level strategic games to obtain daily necessary DSO capacity

The strategic interactions between a DSO and its adjacent prosumage and conventional generators that arise in scenarios Autarky, KfW policy, and DSOwise policy imply a two-level game structure, which can be formulated as an MPEC. The coordination problem that then arises between the different strategic DSOs constitutes an Equilibrium Problem with Equilibrium Constraints (EPEC). 
Recent research in the computational operations research literature has examined the application of numerical solution methods to EPECs (see, e.g., Hu and Ralph, 2007, Siddiqui, 2011, Leyffer and Munson, 2010, Ruiz et al., 2012) but to date only to small-scale problems (Zerrahn and Huppmann, 2017, Huppmann and Siddiqui, 2015). Given the large size of our problem, we choose to simplify our game structure, which allows us to derive numerical results. The following paragraphs give a short description of the steps implemented to [1] derive separated MPECs from EPEC, [2] solve the MPECs for the DSO-wise policy, KfW policy and Autarky scenarios, and [3] refine the results for the DSOwise policy scenario. A comprehensive description can be found in Appendix A.

[1] Derive separated MPECs from an EPEC

To reduce complexity, we abstain from solving the inter-DSO coordination problem by fixing all imports into a DSO region $i m_{n, t}^{T S O}$ to values obtained in the Smart scenario and by adjusting the generation parameters $\bar{G}_{n, t}^{G E N}$ and $C_{n, t}^{G E N}$. The remaining individual MPEC of each DSO are set up by adding the first-order optimality conditions (FOCs) of the adjacent generator and prosumage to the DSO's problem. The derived problem is nonlinear in its objective and its constraints due to complementarity slackness. The following equations give the MPEC formulation for each DSO for a single day computation of the DSO-wise policy scenario. The formulation can be transferred to the $K f W$ policy and Autarky scenarios by parameterizing $\forall n: \alpha_{n}=0.5$ and $\forall n: \alpha_{n}=0$, respectively.

$\min _{\substack{\operatorname{var}^{P R S}, d u^{P R S} \\ \operatorname{var}^{G E N}, u^{G E N} \\ \operatorname{var}^{D S O}}} o b j_{n}^{D S O}=\bar{f}_{n, \tau}^{D S O} \cdot M C^{D S O} \cdot\left|\mathcal{T}_{\tau}\right|+\sum_{t \in \mathcal{T}_{\tau}}\left(1-\alpha_{n}\right) \cdot \bar{G}_{n}^{P R S} \cdot \lambda_{n, t}^{\alpha}, \forall n, \tau$

s.t.

$(2)-(9),(13),(14),(A .2),(A .11)-(A .22) \mid t \in \mathcal{T}_{\tau}$, 
where (13), (14) are the DSO constraints, (2)-(9) are the prosumage constraints, (A.2) is the TSO nodal balance with fixed imports, (A.11) is the adjusted generation constraint, (A.12)-(A.21) are the prosumage FOCs and (A.22) is the generation FOC.

[2] Solve MPECs for scenarios Autarky, KfW policy and DSO-wise policy as mixed-integer linear problems (MILP) using disjunctive constraints formulation

We solve the MPECs as mixed-integer linear problems (MILP). We obtain the MILP by linearizing the complementary slackness using disjunctive constraints (Fortuny-Amat and McCarl, 1981) and through a discretization of the feasible realizations of $\alpha_{n}$. The MILP yields globally optimal results for the predefined discrete values of $\alpha_{n}$ (Conforti et al., 2014). For the scenarios Autarky and KfW policy this is fully equivalent to the nonlinear version of the MPEC as the feasible realization for $\alpha_{n}$ is covered by the discretized values. Yet, for DSO-wise policy, the feasible values of $\alpha_{n}$ are continuous.

[3] Solve MPECs for scenario DSO-wise policy as continuous non-linear problems (NLP)

For DSO-wise policy we solve the original non-linear problem (NLP) version of the MPEC, which yields locally optimal solutions. By initializing the NLP with the result obtained from the MILP, we guarantee that the NLP solution is superior to the globally optimal but discretized outcome from the MILP.

\subsection{Recompute all scenarios with the maximum realized daily DSO capacities} as minimum capacity

Applying this solution strategy, we derive the state-wise necessary DSO capacities for every single day $\bar{f}_{n, \tau}^{D S O}$. Yet, the DSO can not change the provided capacity on a daily basis. Instead, it must supply sufficient capacity for the day of the highest capacity requirement throughout the entire year. 


$$
\bar{f}_{n}^{D S O}=\max _{\tau} \bar{f}_{n, \tau}^{D S O}, \forall n
$$

As a consequence, on many days with previously lower capacity requirements, the available capacity will become abundant. In turn, this may also alter the optimal choice of other variables if there was a trade-off between operational and capacity expenditures in the previous day-to-day calculation. To account for this, we recompute all scenarios holding the DSO capacity fixed at $\bar{f}_{n}^{D S O} \cdot 14$

We implement the problems in GAMS and use the commercial solvers CONOPT for NLP and CPLEX for MILP. Computation time is about $30 \mathrm{~h}$ for the scenario simulations of one year (System: quad-core CPU $2.8 \mathrm{GHz}, 16 \mathrm{~GB}$ RAM).

\section{Results and discussion}

The following section presents modeling results and discusses possible implications. First, we focus on one particular day and one region to detail the general mechanisms that drive the effects of the different policy options on prosumage storage dispatch. We find that the DSO capacity requirement is especially high during the morning hours when PV generation starts to ramp up while pricedriven storage is discharging. Subsequently, we compare the efficiency of the policies examined for different DSO networks. Even though the outcomes of the policy mechanisms are largely consistent overall, quantitative results on capacity reduction potential and necessary compensation differ substantially. Looking at daily required DSO link capacity aggregated across regions, we find that storage exerts ambiguous effects that depend crucially on the policy choice. While smart operation of storage reduces capacity needs, these are increased if

\footnotetext{
${ }^{14}$ The scenarios Smart, No storage and No policy, KfW policy and Autarky can be computed as before with fixed DSO capacities. For DSO-wise policy, it is now also sufficient to compute the MILP as we can obtain a fixed value for $\alpha_{n}$ for the entire year from the necessary capacity.
} 
no policies are implemented. Feed-in policies are also effective in reducing capacity requirements but reach their limit at the point where loads dominate the grid needs. Another important cost driver consists in operational restrictions, which increase system costs for No storage and Autarky in particular. Finally, we evaluate effects on the electricity system level and distributional implications for different players. Compared to no policy, simple feed-in policies are able to close the gap towards a minimum-cost system by $50 \%$. While non-prosumage demand and RES generally benefit from storage availability, this result does not hold for the demand under Autarky.

\subsection{Policy mechanisms at the individual DSO level}

To analyze the mechanisms behind the policies, we first focus on the effects on one particular day and region. We chose the weekday with the highest PV generation - Monday, May 25 - and focus here on the results for Bavaria, which has about $13 \mathrm{GW}$ of PV capacity deployed. Figure 4 shows the realized nodal prices, storage dispatch, and flows in the DSO link for each of the scenarios.

Prices for all scenarios show the typical duck curve pattern (California ISO, 2016) with higher prices in the morning and evening demand peaks and depressed prices during the day due to PV feed-in. The No storage scenario exhibits the most pronounced peaks and valleys. When storage is introduced into such a system, it allows for inter-temporal shifting and thereby reduces price extremes (peaks and valleys are less pronounced in all other scenarios). Prices as well as storage operation are similar for the scenarios Smart, No policy, KfW policy and DSO-wise policy, and differences mainly arise during few hours when the DSO capacities are highly stressed. Interestingly, this is not necessarily the case during times of very high PV generation, as the associated low prices can provide sufficient incentives for market-driven prosumage storage to be charged, and thus mitigate high feed-ins. Instead, differences occur during the morning hours at about 8-9 AM. Here, PV generation already exceeds a third of the daily peak but prices are still relatively high. Therefore, in a scenario in which grid stress is disregarded (No policy), storage is further discharged, leading to addi- 

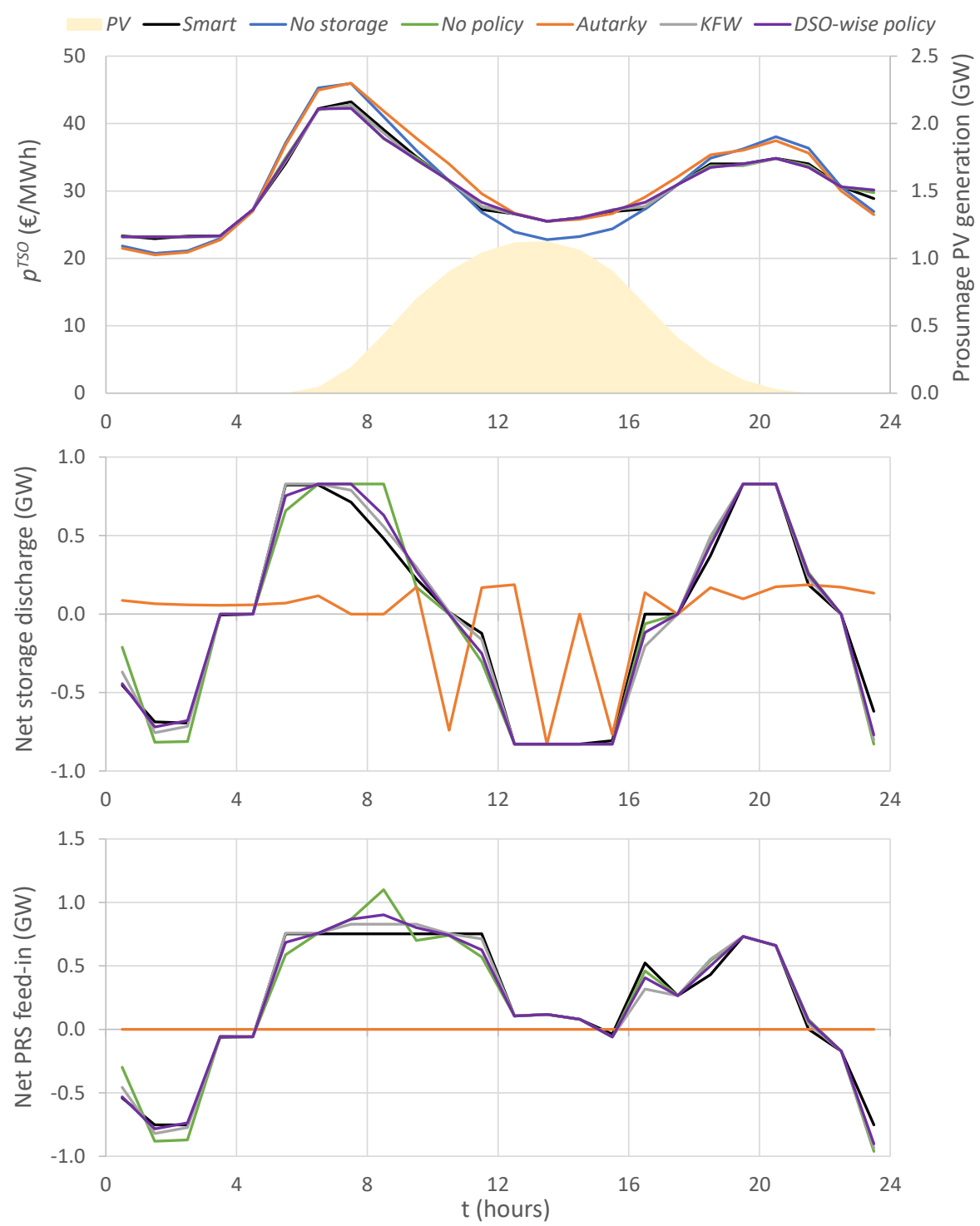

Figure 4: Price curves, prosumage PV generation, storage net discharge, and flow on the DSO link for May 25 in Bavaria. With exception of the Autarky scenario, storage charges at low price hours. The discharge during periods of high prices is influenced by the choice of scenario and crucially affects the DSO capacity needs. 
tional distribution grid stress. Remarkably, in this scenario, peak grid feed-in is even higher than in the No Storage scenario. Those scenarios that take the DSO capacities into account (Smart, DSO-wise policy, KfW policy) show lower storage discharge for these hours. This, in turn, reduces the feed-in and grid requirements and indicates the effectiveness of the policies.

The Autarky scenario exhibits similar prices to the No storage scenario, except for the mid-day hours, where the price valley is less pronounced due to the prohibition of market feed-in from prosumage. In particular, excess prosumage PV generation that cannot be stored must be curtailed. As a consequence, the storage utilization is much lower than in the other storage scenarios and not driven by price differentials like in the other scenarios. Lastly, it is the only scenario without any market interaction. In addition to the prohibition of feedin, also no purchase from the market is needed for the day considered, such that prosumage is fully self-sufficient and does not need any DSO capacity. However, this picture changes for days with lower PV generation.

\subsection{Comparing results for different DSO networks}

Next, we compare results between different states, which resemble separate DSO networks. The optimal trade-off between flexibility provided by the prosumage storage and required DSO capacity is driven by the local share of prosumage in residential demand, local generation patterns, weather conditions, and transmission grid characteristics. Figure 5 shows the maximum allowable feed-in shares $(\alpha)$ for the Smart, No policy, KfW policy, and DSO-wise policy scenarios and induced compensation payments for the latter two. For the majority of states, the required feed-in capacity is lowest in the Smart scenario, followed by KfW policy, DSO-wise policy, and the No policy scenario. Hence, the qualitative pattern that was described above for Bavaria generally holds. ${ }^{15}$ Quantitatively, however, there are great differences among the states and thus

\footnotetext{
${ }^{15}$ Except for the small state Bremen, in which load rather than feed-in is the determining factor.
} 
among the different DSOs. Particularly in states with high demand, high PV generation, and high prosumage shares (BW, BY), DSOs need to provide relatively high compensation to reduce prosumage feed-in. In other large states that have lower PV deployment and prosumage shares (NI, NRW), the compensation is also smaller. Also the policy's effectiveness for different DSOs varies. While in Thuringia (TH), the feed-in reduction potential is substantial, in Lower Saxony

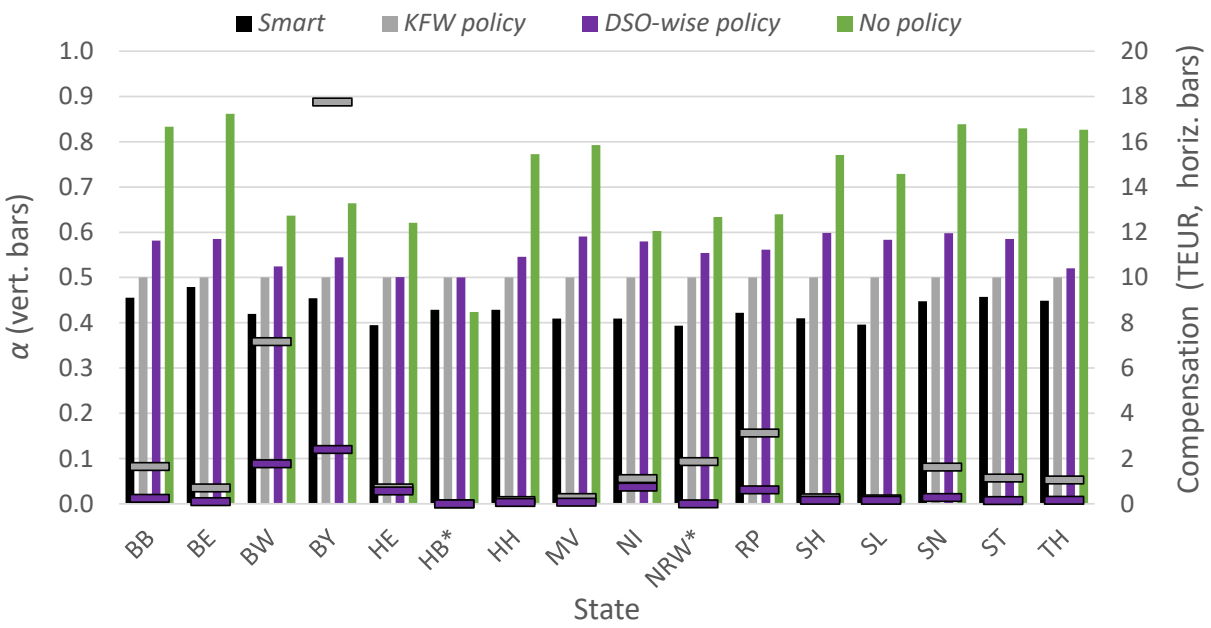

*Load (not feed-in) driven distribution grid stress in DSO-wise policy

Figure 5: State-wise DSO capacity as share of PV peak generation $\left(\alpha_{n}\right)$ and compensation payment for May 25. In the DSO-wise policy policy, $\alpha_{n}$ is greater than for $K f W$ policy in most cases. If distribution stress is solely driven by loads, both policies are ineffective and there is no compensation.

\subsection{Aggregate results on DSO capacities}

With a good understanding of the underlying mechanisms, we can now focus on the policies' aggregate effects on DSO capacity requirements. Figure 6 depicts the sum of daily necessary DSO capacities for all regions throughout the entire year, while Figure 7 gives the share of required DSO capacity which is feedin-driven. ${ }^{16}$ In the benchmark scenario without storage (No storage), we find

\footnotetext{
${ }^{16}$ Note that for the entire year, even larger capacities than the daily maximums might be needed if the state-wise maximums do not coincide.
} 
the highest grid requirement in summer, driven by high PV grid feed-in. In fact, in about half of the cases, DSO capacity is exclusively driven by feed-in in this scenario. Introducing storage into the system substantially decreases required DSO capacities in the scenario Smart. However, the picture changes in the case of purely self-optimizing prosumage (No policy). Here, capacity needs do increase, not only compared to Smart but also in reference to the No storage scenario, and a substantial share of DSO capacity requirements comes as a consequence of feed-ins. Consequently, additional storage capacities can have ambiguous effects on DSO capacities that depend on their mode of operation.

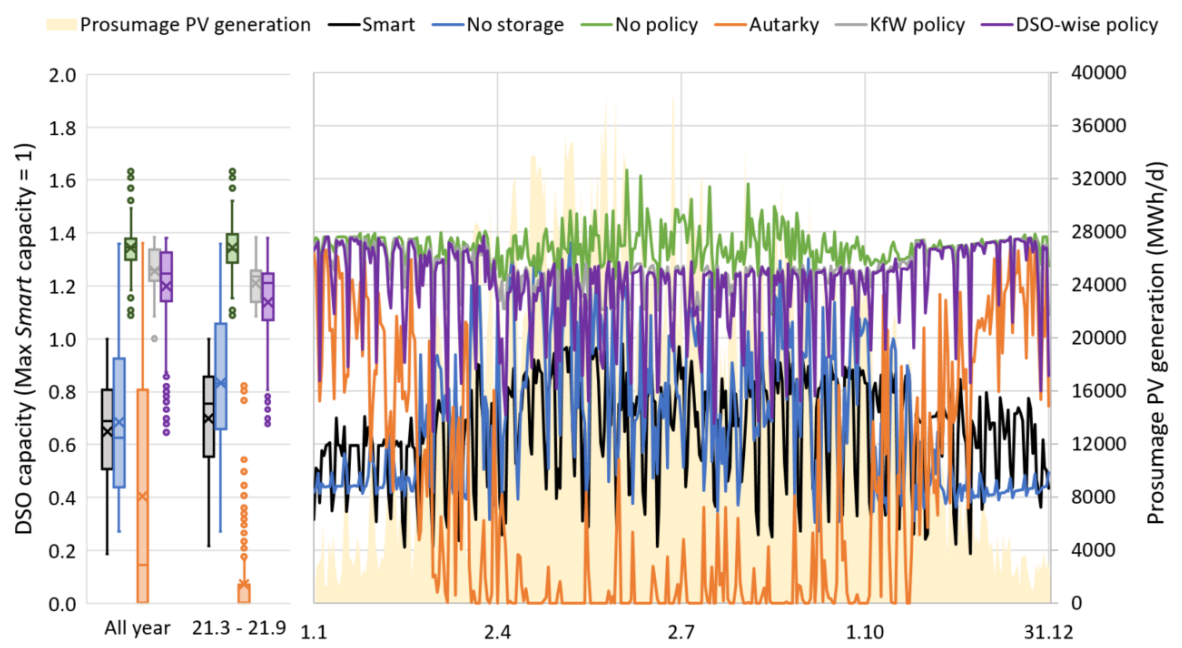

Figure 6: Daily necessary DSO capacity. While in the base case scenarios DSO capacity requirements are PV driven and highest during the summer months, all policy scenarios have lower capacity needs during summer compared to winter. Maximum Smart capacity = $2.6 \mathrm{GW}$.

Looking at the scenarios $K f W$ policy and DSO-wise policy, we find that they are effective in mitigating capacity requirement peaks compared to No policy during the summer months (April-September) with high PV generation. As these account for the highest capacity requirements, the policies manage to reduce the needed capacity. Comparing the two scenarios, the fact that the DSO-wise policy scenario has a higher level of feed-in-induced capacity re- 


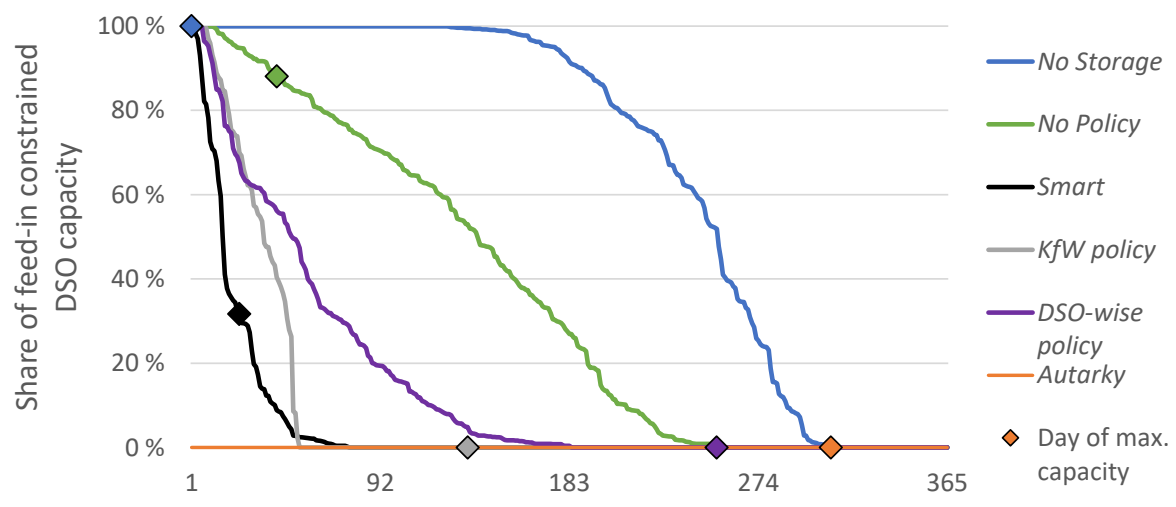

Figure 7: Daily share of feed-in-constrained DSO capacity sorted by size. Day of max (diamond) represents the day of the maximum required DSO capacity.

quirements suggests that the $K f W$ policy policy is overly restrictive. For both scenarios, the remaining grid requirement peak now occurs during the winter months (October-March) and is mainly driven by prosumage load (i.e., flows from the market to the prosumage household) (Figure 7), which the policies cannot affect. As a consequence, also a yearly optimization - as opposed to the day-by-day consideration - could not reduce the needed DSO capacity in the DSO-wise policy or KfW policy scenario.

In the Autarky scenario, DSO capacity requirements are fully driven by load (see Figure 7). During summer, the DSO capacity requirement is reduced substantially, amounting to more than 100 days without any need for DSO capacity. Nevertheless, the scenario is not very effective in reducing load-driven capacity needs during days with very low PV generation as long as seasonal storage is not available. Eventually, about as much DSO capacity has to be deployed as with the other policies or in the No storage scenario.

\subsection{System costs and distributional effects}

To analyze the system-level effects of the different policy options, we look at the change in total system costs of the scenarios as compared to the Smart scenario (Figure 8). From the analysis above, we know that maximum DSO 
capacity requirements associated with the market interactions of prosumage are about the same for the scenarios No storage, KfW policy, DSO-wise policy, and Autarky. Therefore, necessary capacity investment costs compared to the Smart scenario are similar for the four scenarios as well (see Figure 8). However, they differ in the dispatch of generation and storage units, and consequently also in associated operation costs. We find that system costs increase compared to Smart by about a quarter of a percentage point in the scenarios DSO-wise policy and $K f W$ policy. In comparison, with a 0.7 percentage points increase, the rise in costs is more than double in the No policy scenario. For all these scenarios, the increase comes solely from inefficiently high DSO capacities, even despite the slight decrease in operational costs. This phenomenon arises due to the greater operational freedom of prosumage storage (higher DSO capacity allows more market interaction), which leads to a reduction in generation costs due to the consideration of the TSO nodal prices. In the Smart scenario, this freedom is restricted as a means to reduce the requirement of DSO capacities.

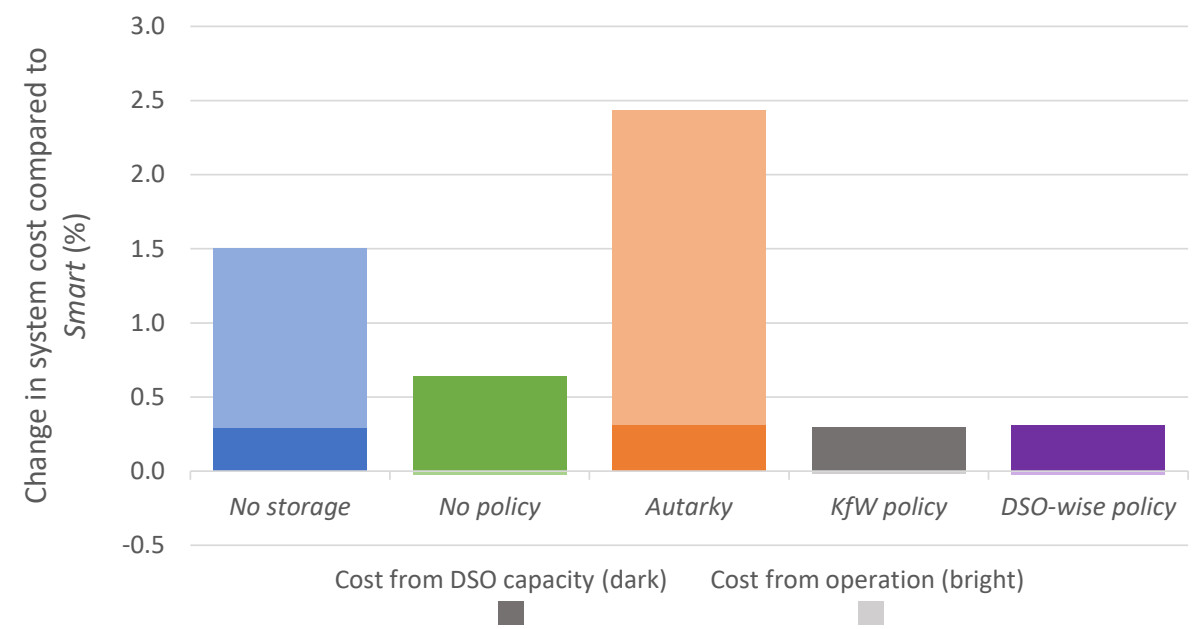

Figure 8: Change in prosumage-induced DSO capacity costs as well as operational costs, which add up to total system costs, compared to the Smart scenario. While DSO capacity costs are particularly high in the No policy scenario, operational costs are even lower than in Smart in the scenarios No policy, KfW policy, and DSO-wise policy but are substantially higher for No storage and Autarky. 
Clearly, the mechanisms change substantially for No storage and the Autarky scenario with its severe restrictions on market interaction. For both of them, operational costs increase substantially. In the No storage scenario, that increase is driven by the fact that expensive peak generation during high demand hours cannot be substituted by cheaper off-peak generation using storage. In the Autarky scenario, storage operation is also heavily restricted. Besides that, excess prosumage PV generation is no longer available to the market, which leads to curtailment and adds to increasing operational costs.

Finally, we assess the beneficiaries and losers in the scenarios. We have seen before that DSO costs from investment are generally similar for the scenarios No storage, Autarky, KfW policy and DSO-wise policy and about $30 \%$ higher than in the Smart scenario. Taking the costs of compensating prosumage into account as well adds another $5 \%$ to the $K f W$ policy scenario, while compensation is negligible for DSO-wise policy (Figure 9). Due to the compensation, prosumage-households are slightly better off under the KfW policy. However, the improvements from the increased operational freedom in the No policy, $K f W$ policy, and DSO-wise policy scenario compared to the Smart scenario are negligible at $<1 \%$. In contrast, prosumage households lose substantially if aiming for Autarky due to lost PV revenues and inefficient storage operation.

Let us now also take a look at non-prosumage demand and renewable generators. We define their objectives as ${ }^{17}$

$$
\begin{aligned}
o b j_{n}^{D} & =\sum_{t}\left(D_{n, t}-l o l_{n, t}^{T S O}\right) \cdot p_{n, t}^{T S O}+l o l_{n, t}^{T S O} \cdot V O L L \\
o b j_{n}^{R E S} & =-\sum_{t}\left(G_{n, t}^{R E S}-c u r t_{n, t}^{T S O}\right) \cdot p_{n, t}^{T S O},
\end{aligned}
$$

Renewable generation and non-prosumage demand both lose in the No storage scenario ( $0.7 \%$ and $0.3 \%$ respectively relative to Smart). This shows that stor-

\footnotetext{
${ }^{17}$ Note that one calculates cost for demand and revenue for RES. Minimizing both terms results in a positive sign for the former and a negative for the latter.
} 
age facilitates renewable capacity deployment (see, e.g., Denholm and Hand, 2011), while conventional generators lose (Sioshansi, 2010). ${ }^{18}$ Renewable generators also gain substantially in the Autarky scenario because the prosumage PV curtailment increases prices, particularly when there is also a substantial amount of generation from non-prosumage PV. From the demand perspective, however, these higher prices induce higher costs. Finally, the two players are relatively indifferent between the scenarios Smart, No policy, KfW policy and DSO-wise policy, with their objectives deviating by well below $0.1 \%$.

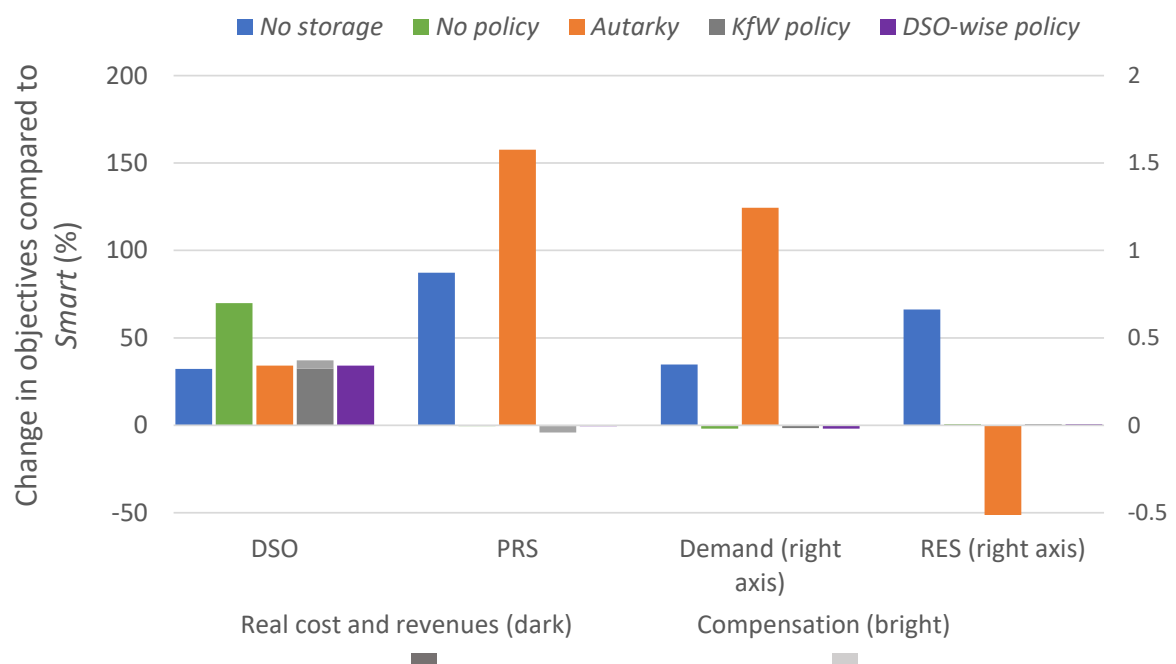

Figure 9: Distributional effects of different scenarios. From our objective definitions (see Eq. 1, 12, 28, 29), positive values indicate a deterioration (less revenues and/or higher costs), while negative values imply improvement for the respective player. Due to compensation (brighter segment of individual bars), prosumage reaps the highest benefits in the KfW policy scenario and loses in the Autarky scenario.

\subsection{Limitations}

Our model setup and calibration relies on some critical assumptions that need to be borne in mind when interpreting the results. For the parametrization

\footnotetext{
${ }^{18}$ Exceptions to this rule may arise, e.g., if storage is owned by oligopolistic generators (Schill and Kemfert, 2011).
} 
of DSO investment costs, we assume unit capacity costs and therefore disregard the economies of scale that are inherent to this infrastructure investment. Taking these into account would reduce system cost differentials between scenarios with high and low distribution grid requirements, while our qualitative results would still hold. Even though we used Germany to calibrate our model, we deviate from some institutional conditions of the national electricity market, such as the single bidding zone. To derive more nuanced estimates of effects of particular policies in a target region, the model can be further adapted to the regulatory settings of the region.

In our representation of storage, we assume that operational conditions do not change over time and do not account for capacity degradation. Reducing the depth of discharge or charging rates can increase battery life (Choi and Lim, 2002) but would also have impacts on the electricity system level. Moreover, we assume that the choice of battery size is independent of the regulatory design, which is aimed at battery dispatch. We leave the assessment of incentives for private storage investment to future research, as this would further complicate the already complex game structure of our setup.

Contrary to small-scale DSO system and prosumage analyses on the individual home or community level, our approach uses a coarse DSO representation but allows us to draw conclusions for a large system. We are aware that our model does not capture all technical aspects of distribution grid management, such as voltage regulation, power factor correction, or the reduction of energy losses (Resener et al., 2018). Moreover, we disregard DSO capacity needed for non-prosumage demand or other small-scale generation. We also abstract from the range of voltage levels handled by DSOs (from the household level at $230 \mathrm{~V}$ to the high-voltage level at $110 \mathrm{kV}$ for Germany) for regional distribution and interconnection. In a trade-off between complexity and tractability, we aggregate the individual components of the distribution grid, comprised, e.g., of distribution lines, transformers, and capacitors. Moreover, our approach simplifies the resulting coordination problem arising among the different DSOs when setting the individual feed-in restriction. With the methods described in Section 7, 
we enforce an equilibrium between the DSOs, but there might well exist other equilibria that we do not explore here.

\section{Conclusions and policy implications}

The increasing number of residential PV systems paired with storage (prosumage) has great potential to benefit the electricity grid as well as the energy system as a whole. Prosumage households provide private capital for both renewable energy and storage deployment, and thus play an important role in the modernization of power systems. As residential battery storage systems become increasingly available and financially viable (Muenzel et al., 2015), the structures of production and storage ownership are inverted, and the technical system characteristics change as well. There is no longer a clear hierarchy, with large conventional generators at high voltage levels and successive transmission and distribution to the consumers. Instead, decentralized generation especially from renewable sources - is fed in along all voltage and grid levels of the system. In this paper, we have analyzed how such storage options can contribute to the integration of RES into a future power system by mitigating distribution grid use and thus facilitating diffusion without the need for grid expansions. Realizing the potential of storage is accompanied by both technical and institutional challenges. To analyze these, we have deployed a comprehensive multi-level capacity planning and dispatch model that mimics the interplay between conventional demand and prosumage, conventional generation and renewables, as well as the DSO and the TSO grid levels. The model accounts for institutional settings and decision-making power of the different players.

Our analysis shows that if storage is deployed without appropriate policies, significant potential system benefits are left untapped. In particular, much of the positive price-moderating effect of storage is eaten up by additional distribution grid requirements. We advise policy makers to provide legal conditions that incentivize prosumage households to operate storage in a system-beneficial manner, e.g., by restricting grid feed-in from PV generation. Simple policies 
like the restriction of maximum grid feed-in based in the nominal PV generation capacity (our $K f W$ policy scenario) are effective in mitigating DSO stress from high prosumage feed-ins. Feed-in policies are, however, ineffective in regulating load-driven DSO stress, e.g., due to high prosumage demand and storage charging from the market at times with low PV generation. As a consequence, even more elaborate feed-in policies cannot further reduce DSO capacity needs substantially. Consequently, complementary load policies are needed, that are able to restrict power purchases from the prosumage household, particularly for charging for storage. In general, we like to caution decision makers when making decisions about storage, as it contributes to both load as well as feedin, and may potentially aggravate both kinds of DSO stresses if no respective operational restrictions or incentives are in place.

Neglecting distribution grid costs (No policy scenario) induces a system cost increase of about $0.6 \%$ compared to a system-optimizing perspective (Smart scenario). This increase can be mitigated by half if simple feed-in policies ( $K f W$ policy, DSO-wise policy) are implemented. Even though these effects are rather small in absolute terms, it is important to note that the changes are all driven by prosumage households, who only contribute about $1 \%$ of total generation and demand. ${ }^{19}$ A higher share of prosumage households will likely induce a respective increase in costs. Significant differences between policy interventions are apparent when looking at the distributional effects on prosumage households and DSOs. While striving for prosumage autarky is more beneficial to the DSO than an unregulated scenario, the prosumage household is worse off. In contrast, policies with incentive payments leave both the DSO and prosumage household better off.

In a nutshell, decision makers should be cautious about the following aspects: 1) Prosumage has a great potential to shape the future power system and facilitate its transition towards sustainability. 2) Nevertheless, prosumage

\footnotetext{
${ }^{19}$ In our parametrization, it is annual prosumage demand: $4.9 \mathrm{TWh}$, prosumage PV generation: 6 TWh, total system demand (incl. PRS): 515 TWh.
} 
may also have adverse effects, such as an associated increase of distribution grid requirements. 3) To tap the full potential of advantages from prosumage, appropriate policies are needed. Feed-in policies can be utilized to partly mitigate grid needs, but must be complemented by load policies to realize the full potential. 4) Careful policy design is vital: otherwise, system costs might even increase with storage.

These findings open up multiple promising avenues for future research: First, this analysis could be extended to include one or multiple load policies. It is likely that even a simple load policy would allow further reductions in DSO capacity needs by addressing cases in which they are driven by peak load in the current scenarios. Also, it opens the option to look at the influence of electric vehicle diffusion, which will likely play a major role in future DSO capacity planning. Moreover, the model can be used to assess the effects of different storage ownership structures (independent vs. prosumage vs. DSO vs. TSO), which may change the incentives for its dispatch and thus imply effects on system operation costs, grid capacity requirements, and distribution of rents. Another promising avenue for further research is the coordination required between different DSOs (a policy set by one DSO may have impacts on prices and thus influence required incentive payments by other DSOs) and the path-dependency that would be implied by any uncoordinated decision making. Furthermore, the incentives arising from the distribution of network charges could be further investigated. This would allow for an investigation of the tradeoff between private storage capacity investment and different possible revenue streams and avoided costs on the prosumage side.

\section{Funding}

Paul Neetzow was supported by the Reiner Lemoine-Stiftung and the German Academic Exchange Service (DAAD). 


\section{Acknowledgements}

The authors would like to thank the participants of the DIW Berlin Brown

Bag Seminar for valuable comments and discussions. Preliminary versions of the paper were also presented and discussed at Young Energy Economists and Engineers Seminar 2017 in Nuremberg, IAEE European Conference 2017 in Vienna and IAEE International Conference 2018 in Groningen.

\section{References}

A.-C. Agricola, B. Höflich, P. Richard, J. Völker, C. Rehtanz, M. Greve, B. Gwisdorf, J. Kays, T. Noll, J. Schwippe, A. Seack, J. Teuwsen, G. Brunekreeft, R. Meyer, and V. Liebert. Ausbau- und Innovationsbedarf der Stromverteilnetze in Deutschland bis 2030. Technical report, Deutsch Energie-Agentur, 2012.

T. Beck, H. Kondziella, G. Huard, and T. Bruckner. Assessing the influence of the temporal resolution of electrical load and pv generation profiles on selfconsumption and sizing of pv-battery systems. Applied energy, 173:331-342, 2016. doi: https://doi.org/10.1016/j.apenergy.2016.04.050.

V. Bertsch, J. Geldermann, and T. Lühn. What drives the profitability of household pv investments, self-consumption and self-sufficiency? Applied Energy, 204:1-15, 2017. doi: https://doi.org/10.1016/j.apenergy.2017.06.055.

BNetzA. Übersicht Strom- und Gasnetzbetreiber, Übersicht Stromnetzbetreiber - Stand: 09.05.2018, 2018. URL https:

//www.bundesnetzagentur.de/DE/Sachgebiete/ElektrizitaetundGas/ Unternehmen_Institutionen/DatenaustauschundMonitoring/ UnternehmensStammdaten/Uebersicht_Netzbetreiber/ UebersichtStromUndGasnetzbetreiber_node.html.

R. E. Bohn, M. Caramanis, and F. Schweppe. Optimal pricing in electrical networks over space and time. RAND Journal of Economics, 15(3):360-376, 1984. 
S. Boyd and L. Vandenberghe. Convex optimization. Cambridge University Press, 2004.

C. Brandstätt, G. Brunekreeft, M. Buchmann, and N. Friedrichsen. Balancing between competition and coordination in smart grids a Common Information Platform (CIP). Economics of Energy 83 Environmental Policy, 6(1), Jan. 2017. ISSN 21605882. doi: 10.5547/2160-5890.6.1.cbra. URL http://www.iaee.org/en/publications/eeeparticle.aspx?id=155.

Bundesverband der Energie- und Wasserwirtschaft e.V. Standardlastprofile, 2015. URL https://www.stromnetz.berlin/en/grid-user.htm.

J. Bushnell. A mixed complementarity model of hydrothermal electricity competition in the western united states. Operations research, 51(1):80-93, 2003.

California ISO. What the duck curve tells us about managing a green grid. Technical report, California Independent System Operator, 2016. URL https://www.caiso.com/Documents/ FlexibleResourcesHelpRenewables_FastFacts.pdf.

J. B. Cardell, C. C. Hitt, and W. W. Hogan. Market power and strategic interaction in electricity networks. Resource and energy economics, 19(1-2): 109-137, 1997.

S. S. Choi and H. S. Lim. Factors that affect cycle-life and possible degradation mechanisms of a li-ion cell based on licoo2. Journal of Power Sources, 111 (1):130 - 136, 2002. ISSN 0378-7753. doi: https://doi.org/10.1016/S03787753(02)00305-1. URL http://www.sciencedirect.com/science/article/ pii/S0378775302003051.

M. Conforti, G. Cornuejols, and G. Zambelli. Integer Programming. Springer, 2014. doi: 10.1007/978-3-319-11008-0.

P. Denholm and M. Hand. Grid flexibility and storage required to achieve very high penetration of variable renewable electricity. Energy Policy, 39(3):18171830, 2011. 
P. Denholm and R. Sioshansi. The value of compressed air energy storage with wind in transmission-constrained electric power systems. Energy Policy, 37 (8):3149-3158, 2009.

A. Dietrich and C. Weber. What drives profitability of grid-connected residential PV storage systems? A closer look with focus on Germany. Energy Economics, 74:399-416, Aug. 2018. ISSN 0140-9883. doi: 10.1016/j.eneco.2018.06.014. URL https://www.sciencedirect.com/ science/article/pii/S0140988318302329.

O. D. M. Dominguez, M. P. Kasmaei, M. Lavorato, and J. R. S. Mantovani. Optimal siting and sizing of renewable energy sources, storage devices, and reactive support devices to obtain a sustainable electrical distribution systems. Energy Systems, pages 1-22, 2017.

J. M. Eyer. Electric utility transmission and distribution upgrade deferral benefits from modular electricity storage: a study for the doe energy storage systems program. Technical report, Sandia National Laboratories, 2009.

L. A. Faerber, N. Balta-Ozkan, and P. M. Connor. Innovative network pricing to support the transition to a smart grid in a low-carbon economy. Energy Policy, 116:210-219, May 2018. ISSN 03014215. doi: 10.1016/ j.enpol.2018.02.010. URL https://linkinghub.elsevier.com/retrieve/ $\mathrm{pii/S0301421518300818.}$

J. Fortuny-Amat and B. McCarl. A representation and economic interpretation of a two-level programming problem. The Journal of the Operational Research Society, 32(9):783-792, 1981. ISSN 01605682, 14769360. URL http://www.jstor.org/stable/2581394.

S. A. Gabriel and F. U. Leuthold. Solving discretely-constrained MPEC problems with applications in electric power markets. Energy Economics, 32(1): 3-14, 2010. 
S. A. Gabriel, A. J. Conejo, J. D. Fuller, B. F. Hobbs, and C. Ruiz. Complementarity Modeling in Energy Markets. Springer, New-York, USA, 2012 edition, 2012. ISBN 1-4419-6122-4. URL http://dx.doi.org/10.1007/978-1-44196123-5.

P. S. Georgilakis and N. D. Hatziargyriou. A review of power distribution planning in the modern power systems era: Models, methods and future research. Electric Power Systems Research, 121:89-100, 2015.

German Federal Ministry for Economic Affairs and Energy. Moderne Verteilernetze für Deutschland (Verteilernetzstudie). Study commissioned by German Federal Ministry for Economic Affairs and Energy, E-Bride Consulting $\mathrm{GmbH}$, Institut für Elektrische Anlagen und Stromwirtschaft (IAEW) der RWTH Aachen, OFFIS Institut für Informatik e.V., 2014. URL http://www.bmwi.de/BMWi/Redaktion/PDF/Publikationen/Studien/ verteilernetzstudie,property=pdf, bereich=bmwi2012, sprache=de,rwb= true.pdf.

M. Graebig, G. Erdmann, and S. Röder. Assessment of residential battery systems (rbs): profitability, perceived value proposition, and potential business models. In 37th IAEE international conference, volume 25, pages 1-15, 2014.

I. Green and R. Staffell. "Prosumage" and the British electricity market. Economics of Energy 83 Environmental Policy, 6(1), Jan. 2017. ISSN 21605882. doi: 10.5547/2160-5890.6.1.rgre. URL http://www.iaee.org/en/ publications/eeeparticle.aspx?id=152.

M. Haller, S. Ludig, and N. Bauer. Bridging the scales: A conceptual model for coordinated expansion of renewable power generation, transmission and storage. Renewable and Sustainable Energy Reviews, 16(5):2687-2695, 2012.

F. Hinz, M. Schmidt, and D. Möst. Regional distribution effects of different electricity network tariff designs with a distributed generation structure: The case of Germany. Energy Policy, 113:97-111, Feb. 2018. ISSN 03014215. 
doi: 10.1016/j.enpol.2017.10.055. URL https://linkinghub.elsevier.com/ retrieve/pii/S0301421517307309.

X. Hu and D. Ralph. Using EPECs to model bilevel games in restructured electricity markets with locational prices. Operations Research, 55(5):809$827,2007$.

D. Huppmann and J. Egerer. National-strategic investment in european power transmission capacity. European Journal of Operational Research, 247(1): 191-203, 2015.

D. Huppmann and S. Siddiqui. An exact solution method for binary equilibrium problems with compensation and the power market uplift problem. Working paper 1475, Deutsches Institut für Wirtschaftsforschung, Berlin (Germany), 2015.

J. Jargstorf, C. De Jonghe, and R. Belmans. Assessing the reflectivity of residential grid tariffs for a user reaction through photovoltaics and battery storage. Sustainable Energy, Grids and Networks, 1:85-98, 2015.

M. Jenabi, S. Fatemi Ghomi, and Y. Smeers. Bi-level game approaches for coordination of generation and transmission expansion planning within a market environment. IEEE Transactions on Power Systems, 28(3):2639-2650, 2013.

K.-P. Kairies, D. Haberschusz, J. van Ouwerkerk, J. Strebel, O. Wessels, D. Magnor, J. Badeda, and D. U. Sauer. Wissenschaftliches Mess-und Evaluierungsprogramm SolarstromspeicherJahresbericht 2016. Technical report, Institut für Stromrichtertechnik und Elektronische Antriebe (ISEA), RWTH Aachen, 2016. URL http://www.speichermonitoring.de/fileadmin/user_upload/ Speichermonitoring_Jahresbericht_2016_Kairies_web.pdf.

KfW. Merkblatt Erneuerbare Energien - KfW-Programm Erneuerbare Energien "Speicher", 2016. URL https://www.kfw.de/Download-Center/ 
F\%C3\%B6rderprogramme-(Inlandsf\%C3\%B6rderung)/PDF-Dokumente/

6000002700_M_275_Speicher.pdf.

M. Klobasa and D. Mast. Analyse der netzausbaukosten und der kostenverteilungswirkung. Technical report, Fraunhofer ISI, DIW Berlin, gws, izes, 2014. URL https://www.impres-projekt.de/impres-wAssets/docs/ 2014_08_03_Netzausbaukosten-ImpRES_final.pdf.

K. Knezović, M. Marinelli, P. Codani, and Y. Perez. Distribution grid services and flexibility provision by electric vehicles: A review of options. In Power Engineering Conference (UPEC), 2015 50th International Universities, pages 1-6. IEEE, 2015.

M. Koch, H. Hermann, F. Flachsbarth, and J. Tambke. Erstellung generischer EE-Strom-Einspeisezeitreihen mit unterschiedlichem Grad an fluktuierendem Stromangebot. Technical report, Ökoinstitut, 2016.

M. Kubli. Squaring the sunny circle? on balancing distributive justice of power grid costs and incentives for solar prosumers. Energy Policy, 114:173-188, 2018.

F. Kunz, J. Weibezahn, P. Hauser, S. Heidari, W.-P. Schill, B. Felten, M. Kendziorski, M. Zech, J. Zepter, C. von Hirschhausen, D. Möst, and C. Weber. Reference Data Set: Electricity, Heat, and Gas Sector Data for Modeling the German System, Dec. 2017. URL https://doi.org/10.5281/ zenodo.1044463.

S. Leyffer and T. Munson. Solving multi-leader-common-follower games. Optimisation Methods \& Software, 25(4):601-623, 2010.

J. López Prol and K. W. Steininger. Photovoltaic self-consumption regulation in Spain: Profitability analysis and alternative regulation schemes. Energy Policy, 108:742-754, Sept. 2017. ISSN 03014215. doi: 10.1016/ j.enpol.2017.06.019. URL https://linkinghub.elsevier.com/retrieve/ pii/S0301421517303750. 
R. Luthander, J. Widén, D. Nilsson, and J. Palm. Photovoltaic self-consumption in buildings: A review. Applied Energy, 142:80-94, 2015.

I. MacGill and R. Smith. Consumers or prosumers, customers or competitors? Some Australian perspectives on possible energy users of the future. Economics of Energy $\&$ Environmental Policy, 6(1), Jan. 2017. ISSN 21605882. doi: 10.5547/2160-5890.6.1.imac. URL http://www.iaee.org/en/ publications/eeeparticle.aspx?id=153.

J. Moshövel, K.-P. Kairies, D. Magnor, M. Leuthold, M. Bost, S. Gährs, E. Szczechowicz, M. Cramer, and D. U. Sauer. Analysis of the maximal possible grid relief from pv-peak-power impacts by using storage systems for increased self-consumption. Applied Energy, 137:567-575, 2015.

V. Muenzel, I. Mareels, J. de Hoog, A. Vishwanath, S. Kalyanaraman, and A. Gort. Pv generation and demand mismatch: Evaluating the potential of residential storage. In Innovative Smart Grid Technologies Conference (ISGT), 2015 IEEE Power \& Energy Society, pages 1-5. IEEE, 2015.

Navigant Research. Global annual deployments of residential energy storage systems are expected to increase from 94.9 MW in 2016 to 3,773.3 MW in 2025, 2016. URL https://www.navigantresearch.com/newsroom/globalannual-deployments-of-residential-energy-storage-systems-areexpected-to-increase-from-94-9-mw-in-2016-to-3773-3-mw-in2025. Press release.

P. Neetzow, A. Pechan, and K. Eisenack. Electricity storage and transmission: Complements or substitutes? Energy Economics. Under review.

Open Power System Data. OPSD data package renewable power plants, 2018. URL https://data.open-power-system-data.org/ renewable_power_plants/2018-03-08/.

I. J. Pérez-Arriaga, J. D. Jenkins, C. Batlle, et al. A regulatory framework for 
an evolving electricity sector: Highlights of the mit utility of the future study. Economics of Energy \& Environmental Policy, 6(1), 2017.

M. Resch, J. Buehler, M. Klausen, and A. Sumper. Impact of operation strategies of large scale battery systems on distribution grid planning in germany. Renewable and Sustainable Energy Reviews, 74:1042-1063, 2017.

M. Resener, S. Haffner, L. A. Pereira, and P. M. Pardalos. Optimization techniques applied to planning of electric power distribution systems: a bibliographic survey. Energy Systems, pages 1-37, 2018.

S. Ruester, S. Schwenen, C. Batlle, and I. Pérez-Arriaga. From distribution networks to smart distribution systems: Rethinking the regulation of European electricity DSOs. Utilities Policy, 31:229-237, 2014.

C. Ruiz, A. Conejo, and Y. Smeers. Equilibria in an oligopolistic electricity pool with stepwise offer curves. IEEE Transactions on Power Systems, 27(2): 752-761, 2012.

W.-P. Schill and C. Kemfert. Modeling strategic electricity storage: The case of pumped hydro storage in germany. The Energy Journal, 32(3):59-87, 2011.

W.-P. Schill, A. Zerrahn, and F. Kunz. Prosumage of solar electricity: pros, cons, and the system perspective. Economics of Energy $\mathscr{E}$ Environmental Policy, 6(1):1-25, 2017. doi: 10.5547/2160-5890.6.1.wsch.

O. Schmidt, A. Hawkes, A. Gambhir, and I. Staffell. The future cost of electrical energy storage based on experience rates. Nature Energy, 2(8):17110, 2017.

F. C. Schweppe, M. C. Caramanis, R. D. Tabors, and R. E. Bohn. Spot Pricing of Electricity. Springer, Berlin, Germany, 1988.

S. Siddiqui. Solving Two-Level Optimization Problems with Applications to Robust Design and Energy Markets. Dissertation at University of Maryland. College Park, USA, 2011. URL https ://drum.lib.umd.edu/handle/1903/12255. Dissertation. University of Maryland. 
R. Sioshansi. Welfare impacts of electricity storage and the implications of ownership structure. The Energy Journal, 31(2):173-198, 2010.

R. Sioshansi. When energy storage reduces social welfare. Energy Economics, 41:106-116, 2014.

J. Solano, M. Brito, and E. Caamao-Martn. Impact of fixed charges on the viability of self-consumption photovoltaics. Energy Policy, 122:322-331, Nov. 2018. ISSN 03014215. doi: 10.1016/j.enpol.2018.07.059. URL https:// linkinghub.elsevier.com/retrieve/pii/S0301421518305056.

P. M. Sotkiewicz and J. M. Vignolo. Nodal pricing for distribution networks: Efficient pricing for efficiency enhancing dg. IEEE Transactions on Power Systems, 21(2):1013-1014, May 2006. ISSN 0885-8950. doi: 10.1109/TPWRS.2006.873006.

K. Spiliotis, A. I. R. Gutierrez, and R. Belmans. Demand flexibility versus physical network expansions in distribution grids. Applied Energy, 182:613$624,2016$.

Umweltbundesamt. Entwicklung des stromverbrauchs nach sektoren in terawattstunden, 2017. URL https://www.umweltbundesamt.de/sites/ default/files/medien/384/bilder/dateien/2_datentabelle-zurabb_entw-stromverbrauch_2017-02-17.pdf.

V. Virasjoki, P. Rocha, A. Siddiqui, and A. Salo. Market impacts of energy storage in a transmission-constrained power system. IEEE Transactions on Power Systems, 31(5):4108-4117, 2016.

Y. Wang, Y. Dvorkin, R. Fernandez-Blanco, B. Xu, T. Qiu, and D. Kirschen. Look-ahead bidding strategy for energy storage. IEEE Transactions on Sustainable Energy, 2017.

H. J. J. Yu. A prospective economic assessment of residential PV selfconsumption with batteries and its systemic effects: The French case in 
2030. Energy Policy, 113:673-687, Feb. 2018. ISSN 03014215. doi: 10.1016/ j.enpol.2017.11.005. URL https://linkinghub.elsevier.com/retrieve/ pii/S0301421517307437.

A. Zerrahn and D. Huppmann. Network expansion to mitigate market power. Networks and Spatial Economics, 17(2):611-644, 2017.

\section{Appendix A. Solution strategy for policy scenarios}

The following section provides detailed descriptions on our approach to solving the three policy scenarios and thus complements Section 7. First, we describe how the inter-DSO coordination problem is reduced to separate problems of the respective DSOs and the adjacent prosumage household and generator at each TSO node (Appendix A.1). Then we show how these problems can be linearized to yield globally optimal results for the discretized solution space (Appendix A.2). Finally, we use these results to initialize the continuous non-linear problem (Appendix A.3).

Appendix A.1. Derive separated MPECs from EPEC

As discussed in Section 3, the problem of finding an equilibrium not only within a DSO region but also between the DSOs requires us to solve an Equilibrium Problem under Equilibrium Constraints (EPEC). To reduce complexity, we enforce the between-DSO equilibrium by fixing imports and exports between DSO networks, thus separating the DSO problems. The remaining Stackelberg game between one DSO and the respective adjacent prosumage and conventional generation can then be characterized by a Mathematical Program under Equilibrium Constraints (MPEC). We set up the state-wise MPEC by adding the prosumage and conventional generation first-order optimality conditions, representing the lower level of the Stackelberg game, to the respective DSO problem.

To allow DSOs a realistic assessment of the effect of their policy intervention on market prices $\left(p_{n, t}^{T S O}\right)$, we add the respective TSO nodal balance to their 
problem. However, we fix imports to values obtained in the Smart scenario, which we denote by $I M_{n, t}^{T S O}$. Moreover, we adjust the parameters of the respective conventional generator to mimic the reaction of the entire conventional generation fleet, rather than only accounting for adjacent conventional generation. We do this by (i) first, introducing an adjusted generation cost parameter $\hat{C}_{n, t}^{G E N}\left(\forall n, t: \hat{C}_{n, t}^{G E N}=p_{n, t}^{T S O} / g_{n, t} \mid g_{n, t}>0\right)$. Here, $p_{n, t}^{T S O}$ and $g_{n, t}$ are taken from results of the Smart scenario. ${ }^{20}$ (ii) Second, we increase the state-wise available generation capacity by $10 \%$, i.e., $\forall n: \hat{\bar{G}}_{n, t}^{G E N}=1.1 \cdot \bar{G}_{n, t}^{G E N}$. These additional local capacities mimic the option to increase imports from the TSO grid.

This setup now allows the DSO to approximate the effects of its decisions on TSO nodal prices and thus on dispatch of prosumage and conventional generation without the need for further coordination between the DSOs. In turn, market prices are the key determinant of potential compensation payments. As a consequence, the solution that we obtain does not fully satisfy the optimality of the between-DSO coordination game. But our parameter adjustment provides a good approximation while substantially reducing complexity and allowing us to obtain numerical results.

In the following, we detail the individual components of the resulting MPEC. For convenience, we use slack variables denoted by $s$ for the lower level first-order optimality conditions to write inequalities as equalities.

$$
\min _{\substack{\operatorname{var}^{P R S}, d u^{P R S} \\ \operatorname{var}^{G E N}, d u^{G E N} \\ \operatorname{var}^{D S O}, s}} \operatorname{obj} j_{n}^{D S O}=\bar{f}_{n}^{D S O} \cdot M C^{D S O} \cdot|\mathcal{T}|+\sum_{t}\left(1-\alpha_{n}\right) \cdot \bar{G}_{n}^{P R S} \cdot \lambda_{n, t}^{\alpha}
$$

s.t.

\footnotetext{
${ }^{20}$ Note that $p_{n, t}^{T S O}=\lambda_{n, t}^{\bar{G}}+C_{n, t}^{G E N} \cdot g_{n, t}$. If generation in a region is at its capacity limit there is a positive price mark-up $\lambda_{n, t}^{\bar{G}} \geq 0$, which reflects the price differential to other regions. Otherwise, if $\lambda_{n, t}^{\bar{G}}=0$, then $\hat{C}_{n, t}^{G E N}=C_{n, t}^{G E N}$.
} 
(13), (14)

$$
\begin{aligned}
& 0=-I M_{n, t}^{T S O}+m 2 d_{n, t}+m 2 s_{n, t}-s 2 m_{n, t}-p v 2 m_{n, t}-g_{n, t}+R_{n, t} \\
& +\operatorname{curt}_{n, t}^{T S O}-\operatorname{lol}_{n, t}^{T S O} \quad\left(p_{n, t}^{T S O}\right) \\
& 0=p v \mathcal{2} d_{n, t}+s \mathcal{2} d_{n, t}+m \mathscr{2} d_{n, t}+\operatorname{lol}_{n, t}^{P R S}-D_{n, t}^{P R S} \quad\left(\lambda_{n, t}^{D}\right) \\
& 0=-p v 2 d_{n, t}-p v 2 s_{n, t}-p v 2 m_{n, t}-\operatorname{curt}_{n, t}^{P R S}+G_{n, t}^{P R S} \quad\left(\lambda_{n, t}^{P V}\right) \\
& 0=\left\{\begin{array}{l}
-s 2 d_{n, t}+\eta \cdot m 2 s_{n, t}-s 2 m_{n, t}+\eta \cdot p v 2 s_{n, t}-e_{n, t}^{P R S}+E_{n}^{P R S}, \text { if } t=1 \\
-s 2 d_{n, t}+\eta \cdot m 2 s_{n, t}-s 2 m_{n, t}+\eta \cdot p v 2 s_{n, t}-e_{n, t}^{P R S}+e_{n, t}^{P R S}, \text { otherwise }
\end{array}\right. \\
& \left(\lambda_{n, t}^{S T O R}\right) \\
& 0=e_{n, t}^{P R S}-\bar{E}_{n}^{P R S}-s_{n, t}^{\bar{E}} \quad\left(\lambda_{n, t}^{\bar{E}}\right) \\
& 0=m 2 s_{n, t}+p v 2 s_{n, t}-\bar{P}_{n}^{P R S}-s_{n, t}^{C H A R G E} \quad\left(\lambda_{n, t}^{C H A R G E}\right) \\
& 0=s 2 d_{n, t}+s 2 m_{n, t}-\bar{P}_{n}^{P R S}-s_{n, t}^{D I S C H} \quad\left(\lambda_{n, t}^{D I S C H}\right) \\
& 0=e_{n t, T}^{P R S}-E_{n}^{P R S} \quad\left(\lambda_{n}^{E}\right) \\
& 0=\alpha_{n} \cdot \bar{G}_{n}^{P R S}-s 2 m_{n, t}-p v 2 m_{n, t}-s_{n, t}^{\alpha} \quad\left(\lambda_{n, t}^{\alpha}\right) \\
& 0=g_{n, t}-\hat{\bar{G}}_{n, t}^{G E N}-s_{n, t}^{\bar{G}} \quad\left(\lambda_{n, t}^{\bar{G}}\right) \\
& 0=\lambda_{n, t}^{P V}-\lambda_{n, t}^{D}-s_{n, t}^{p v 2 d} \quad \perp p v 2 d_{n t, t} \geq 0 \\
& 0=-\lambda_{n, t}^{D}+\lambda_{n, t}^{S T O R}+\lambda_{n, t}^{D I S C H}-s_{n, t}^{s 2 d} \quad \perp s 2 d_{n, t} \geq 0 \\
& 0=p_{n, t}^{T S O}-\lambda_{n, t}^{D}-s_{n, t}^{m 2 d} \quad \perp m 2 d_{n, t} \geq 0 \\
& 0=p_{n, t}^{T S O}-\eta \lambda_{n, t}^{S T O R}+\lambda_{n, t}^{C H A R G E}-s_{n, t}^{m 2 s} \quad \perp m 2 s_{n, t} \geq 0 \\
& 0=-p_{n, t}^{T S O}+\lambda_{n, t}^{S T O R}+\lambda_{n, t}^{D I S C H}+\lambda_{n, t}^{\alpha}-s_{n, t}^{s 2 m} \quad \perp s 2 m_{n, t} \geq 0 \\
& 0=\lambda_{n, t}^{P V}-\eta \lambda_{n, t}^{S T O R}+\lambda_{n, t}^{C H A R G E}-s_{n, t}^{p v 2 s} \quad \perp p v 2 s_{n, t} \geq 0 \\
& 0=-p_{n, t}^{T S O}+\lambda_{n, t}^{P V}+\lambda_{n, t}^{\alpha}-s_{n, t}^{p v 2 m} \quad \perp p v 2 m_{n, t} \geq 0 \\
& 0=\left\{\begin{array}{l}
\lambda_{n, t}^{S T O R}-\lambda_{n}^{E}+\lambda_{n, t}^{\bar{E}}-s_{n, t}^{e}, \text { if } t=T \\
\lambda_{n, t}^{S T O R}-\lambda_{n, t+1}^{S T O R}+\lambda_{n, t}^{E}-s_{n, t}^{e}, \text { otherwise }
\end{array} \quad \perp e_{n, t} \geq 0\right. \\
& 0=-\lambda_{n, t}^{D}+V O L L-s_{n, t}^{l o l} \quad \perp l_{o l}, t \geq 0 \\
& 0=\lambda_{n, t}^{P V}-s_{n, t}^{\text {curt }} \quad \perp \text { curt }_{n, t} \geq 0
\end{aligned}
$$


$0=-p_{n, t}^{T S O}+\hat{C}_{n}^{G E N} g_{n, t}^{G E N}+\lambda_{n, t}^{\bar{G}}-s_{n, t}^{g} \quad \perp g_{n, t}^{G E N} \geq 0$.

Here A.1 is the objective function of an individual DSO, while (13), (14) are the DSO constraints. The TSO nodal balance with fixed imports from the previous Smart computation is given in (A.2), (A.3)-(A.10) are the prosumage constraints with slack variables, (A.11) is the adjusted generation constraint, (A.12)-(A.21) are the prosumage FOCs, and (A.22) is the generation FOC.

The problem is non-linear in its objective (A.1) and due to complementarity slackness. To solve this problem, we first reformulate the MPEC as a mixedinteger linear problem (MILP, see Appendix A.2). The MILP yields globally optimal results for the given choice of discrete variables. Subsequently, we use the MILP results to initialize a continuous non-linear complementarity problem (NLP), which yields locally optimal solutions that weakly dominates the MILP results (see Appendix A.3).

Appendix A.2. Setting up and solving MPEC as mixed-integer linear problems $(M I L P)$

To set up the MILP, we linearize (A.1) by allowing the solver to choose one of eleven discrete choices for $\forall n: \alpha_{n} \in\{0,0.1,0.2, \ldots, 1\}$. To realize this, we introduce $\bar{\alpha}_{i}$ and $b i_{n, i}^{\alpha}$, where $i$ is an auxiliary set, $\bar{\alpha}_{i} \in\{0,0.1,0.2, \ldots, 1\}$ and $b i_{n, i}^{\alpha} \in\{0,1\}$ is a binary vector such that $\forall n: \sum_{i} b i_{n, i}^{\alpha}=1$. We change the non-linear (A.1) to the linear formulation

$$
\begin{aligned}
& \min _{\substack{\operatorname{var}^{P R S}, d u^{P R S} \\
\operatorname{var}^{G E N}, d u^{G E N} \\
\operatorname{var}^{D S O}}} \operatorname{obj} j_{n}^{D S O}=\bar{f}_{n}^{D S O} \cdot M C^{D S O} \cdot|\mathcal{T}|+\sum_{i} \operatorname{comp} p_{n, i}^{D S O} \\
&
\end{aligned}
$$

where $\operatorname{comp}_{n, i}^{D S O}$ is a vector that contains the amount of the optimal compensation for the optimal choice of $i$ and zeros for all other $i^{\prime} s$. It is defined from the following set of equations.

$$
\begin{aligned}
& \sum_{t}\left(1-\bar{\alpha}_{i}\right) \cdot \bar{G}_{n}^{P R S} \cdot \lambda_{n, t}^{\alpha}-\operatorname{comp}_{n, i}^{D S O}-\widetilde{\operatorname{comp}}_{n, i}^{D S O} \leq 0 \\
& \operatorname{comp}_{n, i}^{D S O}-M_{n, i}^{\alpha} \cdot b i_{n, i}^{\alpha} \leq 0
\end{aligned}
$$




$$
\begin{aligned}
& \widetilde{\operatorname{comp}}_{n, i}^{D S O}-M_{n, i}^{\alpha} \cdot\left(1-b i_{n, i}^{\alpha}\right) \leq 0 \\
& \sum_{i} b i_{n, i}^{\alpha}=1 \\
& \alpha_{n}=\sum_{i} \bar{\alpha}_{i} \cdot b i_{n, i}^{\alpha}
\end{aligned}
$$

In this formulation $\widetilde{\operatorname{comp}}_{n, i}^{D S O}$ contains the amounts of compensations for all but the optimal choice of $i$ (for the optimal $i, \widetilde{\operatorname{comp}}_{n, i}^{D S O}$ becomes zero). $M_{n, i}^{\alpha}$ resembles an appropriately large constant, which is larger than the maximum hourly compensation in every state. The last equation (A.28) makes the formulation compatible with the previous equations by defining $\alpha_{n}$. This formulation allows us to replace the objective's non-linearity with a linear integer problem.

Next, we linearize the complementary conditions that arise from the FOCs of prosumage and generation. This is done by using a disjunctive constraint formulation (Fortuny-Amat and McCarl, 1981), which we apply to the complementarity conditions of equations (A.6)-(A.8), (A.10), (A.11)-(A.22). Instead of enforcing complementarity with the constraints themselves, we use the respective slack variables to formulate the disjunctive constraints. This replaces the bilinear complementarity conditions with linear integer constraints.

The disjunctive constraints for our problems can be written as:

$$
\begin{aligned}
& s_{n, t}^{\bar{E}}-M_{n, t}^{\bar{E}} \cdot b i_{n, t}^{\bar{E}} \leq 0 \\
& \lambda_{n, t}^{\bar{E}}-M_{n, t}^{\bar{E}} \cdot\left(1-b i_{n, t}^{\bar{E}}\right) \leq 0 \\
& s_{n, t}^{C H A R G E}-M_{n, t}^{C H A R G E} \cdot b i_{n, t}^{C H A R G E} \leq 0 \\
& \lambda_{n, t}^{C H A R G E}-M_{n, t}^{C H A R G E} \cdot\left(1-b i_{n, t}^{C H A R G E}\right) \leq 0 \\
& s_{n, t}^{D I S C H}-M_{n, t}^{D I S C H} \cdot b i_{n, t}^{D I S C H} \leq 0 \\
& \lambda_{n, t}^{D I S C H}-M_{n, t}^{D I S C H} \cdot\left(1-b i_{n, t}^{D I S C H}\right) \leq 0 \\
& s_{n, t}^{\alpha}-M_{n, t}^{\alpha} \cdot b i_{n, t}^{\alpha} \leq 0 \\
& \lambda_{n, t}^{\alpha}-M_{n, t}^{\alpha} \cdot\left(1-b i_{n, t}^{\alpha}\right) \leq 0 \\
& s_{n, t}^{\bar{G}}-M_{n, t}^{\bar{G}} \cdot b i_{n, t}^{\bar{G}} \leq 0 \\
& \lambda_{n, t}^{\bar{G}}-M_{n, t}^{\bar{G}} \cdot\left(1-b i_{n, t}^{\bar{G}}\right) \leq 0
\end{aligned}
$$




$$
\begin{aligned}
& s_{n, t}^{p v 2 d}-M_{n, t}^{p v 2 d} \cdot b i_{n, t}^{p v 2 d} \leq 0 \\
& p v 2 d_{n, t}-M_{n, t}^{p v 2 d} \cdot\left(1-b i_{n, t}^{p v 2 d}\right) \leq 0 \\
& s_{n, t}^{s 2 d}-M_{n, t}^{s 2 d} \cdot b i_{n, t}^{s 2 d} \leq 0 \\
& s 2 d_{n, t}-M_{n, t}^{s 2 d} \cdot\left(1-b i_{n, t}^{s 2 d}\right) \leq 0 \\
& s_{n, t}^{m 2 d}-M_{n, t}^{m 2 d} \cdot b i_{n, t}^{m 2 d} \leq 0 \\
& m 2 d_{n, t}-M_{n, t}^{m 2 d} \cdot\left(1-b i_{n, t}^{m 2 d}\right) \leq 0 \\
& s_{n, t}^{m 2 s}-M_{n, t}^{m 2 s} \cdot b i_{n, t}^{m 2 s} \leq 0 \\
& m 2 s_{n, t}-M_{n, t}^{m 2 s} \cdot\left(1-b i_{n, t}^{m 2 s}\right) \leq 0 \\
& s_{n, t}^{s 2 m}-M_{n, t}^{s 2 m} \cdot b i_{n, t}^{s 2 m} \leq 0 \\
& s 2 m_{n, t}-M_{n, t}^{s 2 m} \cdot\left(1-b i_{n, t}^{s 2 m}\right) \leq 0 \\
& s_{n, t}^{p v 2 s}-M_{n, t}^{p v 2 s} \cdot b i_{n, t}^{p v 2 s} \leq 0 \\
& p v 2 s_{n, t}-M_{n, t}^{p v 2 s} \cdot\left(1-b i_{n, t}^{p v 2 s}\right) \leq 0 \\
& s_{n, t}^{p v 2 m}-M_{n, t}^{p v 2 m} \cdot b i_{n, t}^{p v 2 m} \leq 0 \\
& p v 2 m_{n, t}-M_{n, t}^{p v 2 m} \cdot\left(1-b i_{n, t}^{p v 2 m}\right) \leq 0 \\
& s_{n, t}^{e}-M_{n, t}^{e} \cdot b i_{n, t}^{e} \leq 0 \\
& e_{n, t}-M_{n, t}^{e} \cdot\left(1-b i_{n, t}^{e}\right) \leq 0 \\
& s_{n, t}^{l o l}-M_{n, t}^{l o l} \cdot b i_{n, t}^{l o l} \leq 0 \\
& l o l_{n, t}-M_{n, t}^{l o l} \cdot\left(1-b i_{n, t}^{l o l}\right) \leq 0 \\
& s_{n, t}^{\text {curt }}-M_{n, t}^{\text {curt }} \cdot b i_{n, t}^{\text {curt }} \leq 0 \\
& \text { curt }_{n, t}-M_{n, t}^{\text {curt }} \cdot\left(1-b i_{n, t}^{\text {curt }}\right) \leq 0 \\
& s_{n, t}^{g}-M_{n, t}^{g} \cdot b i_{n, t}^{g} \leq 0 \\
& g_{n, t}-M_{n, t}^{g} \cdot\left(1-b i_{n, t}^{g}\right) \leq 0
\end{aligned}
$$

For all pairs of disjunctive constraints, $M$ resembles an appropriately large constant $^{21}$ and $b i \in\{0,1\}$ a binary variable. For $b i=0$, it is straightforward

\footnotetext{
${ }^{21}$ See, e.g., Gabriel and Leuthold (2010) for a discussion on how to choose appropriate constants.
} 
that $s=0$ and $\lambda \geq 0$. Hence, this indicates a binding constraint. On the other hand, for $b i=1$, it follows that $s \geq 0$ and $\lambda=0$, which thus indicates a non-binding constraint.

These disjunctive constraints finalize the MILP formulation of our MPEC, which is implemented as

Equation $(A .23)$

s.t. (13), (14), (A.2) - (A.22) without complementarities,

$$
(A .24)-(A .60)
$$

To facilitate solving this problem, we initialize the MILP with the solution of the Smart scenario. In doing so, we also incorporate the marginals of the equations (2)-(9), (11), (16) to initialize the explicit dual variables.

Appendix A.3. Setting up and solving the MPEC as a continuous non-linear problem (NLP)

Finally, we use the numerically derived solution to the MILP to initialize a continuous NLP. An NLP cannot guarantee globally optimal solutions, but starting from the discretely optimal solutions of the MILP, the solver would only choose to change the solution if an improvement could be achieved by deviating from the discrete values for $\alpha_{n}$. Hence, the obtained solutions to the NLP weakly dominate the MILP solutions, even if it cannot be guaranteed that they represent the global optima.

To set up the NLP, we write the complementarity slackness conditions in bilinear form instead of the previously used disjunctive formulation.

Equation $(A .1)$

s.t.

(13), (14), (A.2) - (A.22) without complementarities

$s_{n, t}^{\bar{E}} \cdot \lambda_{n, t}^{\bar{E}}=0$

$s_{n, t}^{C H A R G E} \cdot \lambda_{n, t}^{C H A R G E}=0$ 


$$
\begin{aligned}
& s_{n, t}^{D I S C H} \cdot \lambda_{n, t}^{D I S C H}=0 \\
& s_{n, t}^{\alpha} \cdot \lambda_{n, t}^{\alpha}=0 \\
& s_{n, t}^{\bar{G}} \cdot \lambda_{n, t}^{\bar{G}}=0 \\
& s_{n, t}^{p v 2 d} \cdot p v 2 d_{n t, t}=0 \\
& s_{n, t}^{s 2 d} \cdot s 2 d_{n, t}=0 \\
& s_{n, t}^{m 2 d} \cdot m 2 d_{n, t}=0 \\
& s_{n, t}^{m 2 s} \cdot m 2 s_{n, t}=0 \\
& s_{n, t}^{s 2 m} \cdot s 2 m_{n, t}=0 \\
& s_{n, t}^{p v 2 s} \cdot p v 2 s_{n, t}=0 \\
& s_{n, t}^{p v 2 m} \cdot p v 2 m_{n, t}=0 \\
& s_{n, t}^{e} \cdot e_{n, t}=0 \\
& s_{n, t}^{l o l} \cdot l o l_{n, t}=0 \\
& s_{n, t}^{c u r t} \cdot c^{2}=0 \\
& s_{n, t}^{g} \cdot g_{n, t}^{G E N}=0
\end{aligned}
$$

